\title{
Nonradiative and Radiative Recombination in CdS Polycrystalline Structures
}

\author{
E. Gaubas, ${ }^{1}$ V. Borschak, ${ }^{2}$ I. Brytavskyi, ${ }^{2}$ T. Čeponis, ${ }^{1}$ D. Dobrovolskas, ${ }^{1}$ \\ S. Juršènas, ${ }^{1}$ J. Kusakovskij, ${ }^{1}$ V. Smyntyna, ${ }^{2}$ G. Tamulaitis, ${ }^{1}$ and A. Tekorius ${ }^{1}$ \\ ${ }^{1}$ Institute of Applied Research, Vilnius University, Sauletekio Avenue 9-III, LT-10222 Vilnius, Lithuania \\ ${ }^{2}$ Odessa I.I.Mechnikov National University, Dvoryanskaya Street 2, Odessa, Ukraine \\ Correspondence should be addressed to E. Gaubas; eugenijus.gaubas@ff.vu.lt
}

Received 13 May 2013; Revised 27 August 2013; Accepted 27 August 2013

Academic Editor: David Huber

Copyright (C) 2013 E. Gaubas et al. This is an open access article distributed under the Creative Commons Attribution License, which permits unrestricted use, distribution, and reproduction in any medium, provided the original work is properly cited.

Properties of polycrystalline CdS layers, employed in formation of the $\mathrm{CdS}-\mathrm{Cu}_{2} \mathrm{~S}$ heterostructures, have been studied by combining contactless techniques of the time and spectrally resolved photoluminescence (TR-PL) spectroscopy and microwaveprobed photoconductivity (MW-PC) transients. The confocal microscopy has been employed to correlate the homogeneity of photoluminescence and grain size in CdS layers. Three types of samples with crystallite grain size of $<1 \mu \mathrm{m}$ (the I-type) and of $2-$ $10 \mu \mathrm{m}$ of homogeneous (II-type) and inhomogeneous (III-type) grain distribution have been separated. The simultaneous record of MW-PC and TR-PL responses ensures the same sampling area on the layer under investigation, as both (MW-PC and TRPL) signals are generated by the same UV laser excitation beam. Two PL bands peaked at 500 and 700 nm were revealed. It has been demonstrated that photoluminescence intensity strongly depends on the properties of the polycrystalline 15-26 $\mu \mathrm{m}$ thick CdS layers with equilibrium carrier density of about $1.5 \times 10^{13} \mathrm{~cm}^{-3}$, which serve as the substrates to form $\mathrm{CdS}_{-} \mathrm{Cu}_{2} \mathrm{~S}$ junctions. The different carrier decay components were ascribed to different microareas with characteristic MW-PC and PL decay lifetimes of 2-10 ns, ascribed to microcrystallites with PL instantaneous decay lifetimes of 40-200 ns, and MW-PC decay lifetimes in the range of $100-1000 \mu$ s attributed to the inter-crystallite areas of CdS polycrystalline material.

\section{Introduction}

The CdS and $\mathrm{Cu}_{2} \mathrm{~S}$ compounds are of great interest owing to their unique properties in variation of composition and their potential applications in numerous fields $[1,2]$, such as sensor elements in X-ray imaging devices [3], solar cells [4], and super ionic conductors, photodetectors, and photothermal converters [5-9]. Cadmium sulfide CdS is also widely used as a promising material in applications of solar cells $[10,11]$. Much research interest comprises the possible applications of CdS in fabrication of various optoelectronic devices [10] based on CdS polycrystalline films. The CdS films are obtained by various methods to form layers with optimized efficiency, using techniques of deposition in vacuum [11], of thermal evaporation $[12,13]$, sputtering [14], chemical spraying [15], silk screen printing, sintering, chemical printing, and electrophoretic deposition $[4,11,16]$. Formation of CdS$\mathrm{Cu}_{2} \mathrm{~S}$ heterojunction structures is usually implemented by wet dipping of the pre-etched evaporated in vacuum CdS film into a hot solution of $\mathrm{CuCl}$ [17].

In this work, the CdS polycrystalline films, exploited for formation of the $\mathrm{CdS}-\mathrm{Cu}_{2} \mathrm{~S}$ heterojunctions, have been studied. The CdS layer serves as a substrate for deposition of $\mathrm{Cu}_{2} \mathrm{~S}$ emitter. $\mathrm{CdS}$ layer also makes a base region within $\mathrm{CdS}-\mathrm{Cu}_{2} \mathrm{~S}$ heterojunction. The previous study of these CdS$\mathrm{Cu}_{2} \mathrm{~S}$ layered structures show that the parameters of effective doping, of crystalline precipitates, of capacitance characteristics, and of introduced traps spectrum considerably changes $[18,19]$, depending on layer deposition regimes, in respect of thermal and exposure processes. Thus, characterization of the carrier recombination channels and carrier decay rates is important in understanding and manipulation of the junction structures. Intercrystallite volumes of disordered structure within the polycrystalline CdS material cause the effect of photoinduced modulation of junction potential barrier. This determines a percolative carrier transport due 
to a system of disordered distribution of size and location of the microcrystals. The disordered material surrounding volumes of crystallites leads to the stretched exponential relaxation (SER) of the density of excess carriers. Study of these relaxation processes allows one to estimate and to compare the disorder specific parameters of various samples. The radiative and nonradiative recombination channels in crystallites have been examined by simultaneous sampling of the photoconductivity and photoluminescence transients attributed to the same local area within polycrystalline CdS layer. These investigations have been combined with confocal microscopy imaging (CMI) to correlate crystallite size and their density with distribution of the luminescence intensity.

The confocal microscopy imaging (CMI) combined with PL spectroscopy enabled us to correlate the homogeneity of photoluminescence and the grain size in CdS layers. Three types of $15-26 \mu \mathrm{m}$ thick polycrystalline CdS samples with crystallite grain size of $<1 \mu \mathrm{m}$ (the I-type) and of $2-10 \mu \mathrm{m}$ of quasihomogeneous (II-type) and inhomogeneous (III-type) grain distribution have been separated. The simultaneous record of MW-PC and TR-PL responses ensures the same sampling area on the layer under investigation, as both (MW$\mathrm{PC}$ and TR-PL) signals are generated by the same UV laser excitation beam. Two spectral bands of photoluminescence have been observed within all types of samples: a narrower band of green luminescence (G-PL) peaked at $500 \mathrm{~nm}$, which can be associated with interband radiative recombination, and a wider spectral band of red luminescence (R-PL) with a peak at $700 \mathrm{~nm}$, which can be ascribed to deep trap mediated radiative transitions. Duration of the initial MW-PC transient component with instantaneous decay lifetimes of 2-10 ns is in the same time-scale with G-PL decay. The microwave-probed photoconductivity transients with asymptotic instantaneous decay lifetimes in the range of $100-1000 \mu$ s exhibited carrier decay components associated with carrier decay within the disordered material surrounding microcrystals in the CdS polycrystalline layer. The different carrier decay components were ascribed to different microareas: the characteristic MWPC and PL decay lifetimes of 2-10 ns are ascribed to the microcrystallites; the PL instantaneous decay lifetimes of 40$200 \mathrm{~ns}$ and MW-PC decay lifetimes in the range of 100$1000 \mu \mathrm{s}$ are attributed to the intercrystallite areas of CdS polycrystalline material. The R-PL peaked at $700 \mathrm{~nm}$ can be ascribed to centres with activation energy $E_{\mathrm{A}, \mathrm{DL}}=1.75 \mathrm{eV}$ which is in good agreement with that $E_{\mathrm{DL}}=1.65 \pm 0.04 \mathrm{eV}$ extracted from photoionization spectra recorded on the same CdS samples. The observed differences of MW-PC transients have been explained by larger volumes of microcrystals in II- and III-type samples. Owing to the larger volumes (and ratio of bulk material to crystallite surface) of microcrystals in II- and III-type samples, as observed in confocal microscopy images, the radiative (G-PL) interband recombination efficiency is elevated in II- and III-type samples. These observations imply that processes of radiative recombination, during rather long time of about $200 \mathrm{~ns}$, contemporize also in microcrystals surrounding structure by exchange of carriers between microcrystals and their boundary area. This has been explained by excess carrier density accelerated radiative recombination through trap levels, assuming a nonradiative capture of carriers before radiative transition and a barrier modified radiative recombination. It has been deduced that barrier widens and sinks with increase of excess carrier density, and thereby energy of emitted photons decreases. It has been inferred that samples of the II-type and the III-type are structurally similar, and that samples of the I-type possess a distinguishably disordered structure. Spatial inhomogeneity, caused by dislocations and other extended imperfections located within these periphery areas surrounding microcrystals, determines carrier recombination and transport features. Excess carriers, brought to the periphery areas containing space charge regions, are separated by space charge field, and their recombination is only mediated by diffusion, owing to excess carrier density gradients. Carrier capture within local space-charge region fields seems to be modified by potential barrier. Screening of this barrier is dependent on equilibrium and excess carrier density. Thereby, the observed G-PL peak intensity in different type samples and its spectral shifts have been explained by variation of carrier densities in various type polycrystalline structures. Different (fast and slow) carrier decay components should be ascribed to different microareas of $\mathrm{CdS}$ polycrystalline material. It has been confirmed by photoluminescence (PL) microscopy that PL signal contains a granular structure. Thus, the simultaneous control of photoconductivity (ascribed to integrated over excitation beam spot area) and green photoluminescence (associated with interband recombination within microcrystalline volumes) spectrum and time resolved signals can be a tool to resolve an impact of different microvolumes to carrier decay parameters. It has been identified from confocal microscopy imaging that centres of the G-PL and R-PL are spatially separated for the I- and III-type samples, while spatial location of these G-PL and R-PL centres nearly coincides for samples of the II-type. The confocal microscopy images among the CdS polycrystalline layers of different types imply that the most homogeneous $\mathrm{CdS}$ layer is obtained for the II-type samples, deposited at $260^{\circ} \mathrm{C}$ for $75 \mathrm{~min}$. While the PL spectra show that interband recombination within microcrystals (associated with G-PL) prevails in III-type samples, deposited at $260^{\circ} \mathrm{C}$ for $80 \mathrm{~min}$. This is in agreement with pulsed barrier capacitance (BELIV) characteristics [18], where the most qualitative $\mathrm{CdS}-\mathrm{Cu}_{2} \mathrm{~S}$ junctions (with clearly expressed barrier capacitance and a relatively small leakage current) were obtained on CdS substrates of the III-type. A linearization of the MW-PC transient asymptotic component is possible when using a stretched exponent (SER) approximation with fractional index $\alpha$ different for different types of $\mathrm{CdS}$ structures investigated. Values of the fractional index $\alpha$ have been obtained in the range of $\alpha=0.16-0.20$ for different samples. It has been inferred from SER analysis that samples of the II-type and the III-type are structurally similar and are characterized by fractal factor of $f \cong 0.14$, and that samples of the I-type exhibit a distinguishably disordered structure with fractal factor $f \cong 0.17$. Value of free-carrier density of the $n_{0}=1.5 \cdot 10^{13} \mathrm{~cm}^{-3}$ was estimated for junctions of II- and III-type, and it determines concentration of shallow impurities. For the I-type samples, equilibrium carrier density is significantly smaller; thus, junction appeared to be 
fully depleted without external applied voltage. The observed differences of MW-PC transients can be explained by larger volumes of micro-crystallites in II- and III-type samples. The density of deep traps is the smallest in microcrystals of IIItype samples. While, the most homogeneous distribution of microcrystals has been obtained for the II-type samples.

\section{Deposition of Polycrystalline CdS Layers}

CdS layers were deposited by evaporation in vacuum of $\mathrm{CdS}$ powder. The deposition rate was about of $0.3 \mu \mathrm{m} / \mathrm{min}$, and a glass substrate temperature was held in the range of $220-260^{\circ} \mathrm{C}$ during the deposition. The evaporation time was varied from 60 to $80 \mathrm{~min}$ to get a thick enough $(15-26 \mu \mathrm{m})$ CdS layer. The thermal anneal in vacuum at $T=450^{\circ} \mathrm{C}$ for $30 \mathrm{~min}$ was exploited after deposition of CdS layer to perform the recrystallization of the as-deposited CdS. These thermal treatments determine an increase of the crystallite size and a reduction of the crystal defects density [20].

\section{Measurement Techniques}

Study of electrical features, of X-ray diffraction (XRD) patterns [18], and of deep traps level spectroscopy in $\mathrm{CdS}-\mathrm{Cu}_{2} \mathrm{~S}$ structures [19] showed that several types of samples can be separated. These sample types exhibit different characteristics dependent on temperature and duration of layer deposition. These deposition parameters were varied in the range of $220-260^{\circ} \mathrm{C}$ of temperatures and durations of 50-80 min. The preliminary observations of the microwave probed photoconductivity transients (MW-PC) implied that slow MWPC component appears due to carriers brought to periphery of microcrystals within CdS layer. The different (fast and slow) carrier decay components were ascribed to different microareas of CdS polycrystalline material. The obtained differences among samples relative to the electrical, spectral, and temporal characteristics were hypothetically ascribed to lateral inhomogeneity of deposited layers. Therefore, the simultaneous control of photoconductivity (ascribed to integrated over excitation beam spot area) and spectrum as well as time resolved photoluminescence (TR-PL) signals (associated with microcrystalline volumes) have been chosen as a tool to resolve an impact of different layer microvolumes on carrier decay. The simultaneous record of MW-PC and $\mathrm{PL}$ responses ensures the same sampling area on the layer under investigation, as both (MW-PC and TR-PL) signals are generated by the same UV laser excitation beam. The time resolved MW-PC and PL (also spectrally dispersed) measurements were combined with confocal microscopy imaging (CMI) to relate microcrystallite size (from CMI) and PL efficiency (spectral) local variations.

A sketch of experimental arrangement, applied in this work for simultaneous MW-PC and spectrum as well as time resolved PL measurements is shown in Figure 1.

Transient signals of the MW-PC and of PL have been synchronously collected and integrated over the same UV $(354 \mathrm{~nm})$ excitation beam spot. A microchip laser STA-1TH was employed for pulsed (500 ps) ultraviolet (UV) light generation of the excess carriers. Intensity of excitation is varied by spectrally neutral optical filters. The MW-PC response has been detected by using a coaxial needle-tip probe [21]. The registered MW-PC signal is transferred from a microwave detector to a digital $1 \mathrm{GHz}$ oscilloscope TDS5104, equipped with PC computer, where MW-PC transient is displayed and processed. The PL light is collected from the area within a bisector between the incident and reflected UV light beams. The UV filtered PL beam is focused onto a slit of monochromator. The PL light is dispersed using a grating monochromator. The PL pulsed signal is detected by a Hamamatsu H10721 photomultiplier. This detected PL signal is also transferred to another channel of the digital oscilloscope Tektronix TDS-5104, where PL transient is displayed and processed together with MW-PC transient.

The confocal microscopy imaging has been implemented by using UV continuous-wave laser and microscope Alpha 300-WITec. The spatial distribution of PL intensity within a fragment of area of the CdS layer was studied on a submicrometer scale. For spectral resolution, the microscope was coupled by an optical fiber with a UHTS 300 spectrometer followed by a thermoelectrically cooled CCD camera. The excitation beam of a CW He-Cd laser emitting at $442 \mathrm{~nm}$ was focused onto the sample using a high numerical aperture $(\mathrm{NA}=0.9)$ objective. This ensured that the in-plane spatial resolution is approximately $220 \mathrm{~nm}$.

\section{Results and Discussion}

4.1. Preliminary Sorting of the CdS Polycrystalline Structures. A barrier evaluation by pulsed linearly increasing voltage (BELIV) technique [22, 23] was applied to determine the temporal characteristics of the junction barrier charging and carrier generation currents within CdS junction base region. These results are discussed in more detail elsewhere [18, 19], while the main features are summarized below.

Three types of BELIV transients (Figure 2), associated with $\mathrm{CdS}$ polycrystal formation regimes, have been obtained over sets of the investigated in dark $\mathrm{Cu}_{2} \mathrm{~S}-\mathrm{CdS}$ samples. The square-wave shape BELIV transients are inherent for the I-type samples, which were deposited at $T_{\text {dep }}=220^{\circ} \mathrm{C}$ of substrate temperature for $t_{\mathrm{dep}}=60 \mathrm{~min}$. These I-type BELIV transients imply the insulator-specific state of base material, which lacks of free-carriers. The II-type and IIItype samples are similar, as exhibiting the junction, which depletion can be modified by external voltage. The main difference (in respect of the electrical characteristics of these II- and III-type samples) appears in generation current. For the II-type samples $\left(T_{\text {dep }}=260^{\circ} \mathrm{C}, t_{\text {dep }}=75 \mathrm{~min}\right)$, the generation current component is pronounced within the rearward wing of transient due to carrier emission from deep traps. For the III-type samples $\left(T_{\text {dep }}=260^{\circ} \mathrm{C}, t_{\text {dep }}=\right.$ $80 \mathrm{~min}$ ), the barrier charging current peak prevails which is accompanied with the descending current component due to charge extraction. The observed differences over the BELIV transient shapes were explained [18] assuming variation of relative densities of dopants (which form shallow levels) and carrier capture centres (associated with rather deep levels). Value of free-carrier density of more than $10^{13} \mathrm{~cm}^{-3}$ was estimated for junctions of II- and III-type, and it determines 


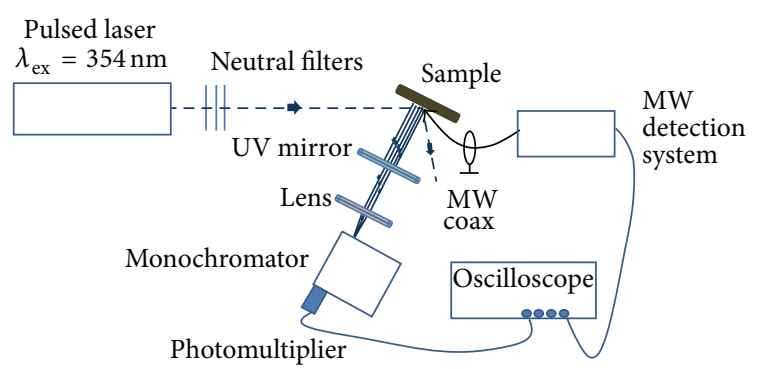

(a)

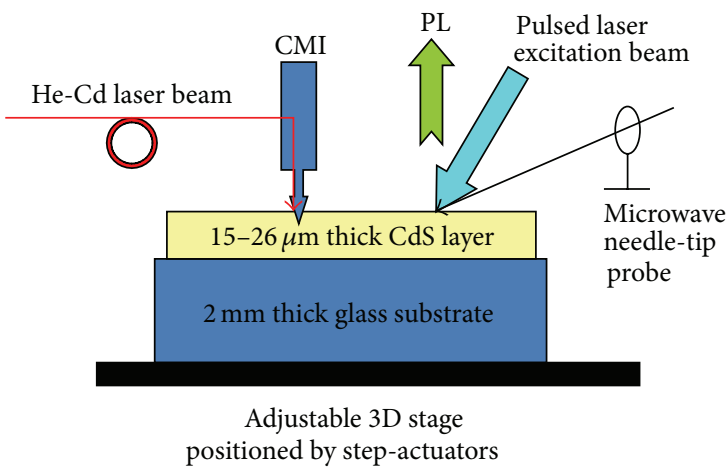

(b)

FIGURE 1: (a) Setup of instrumentation for the simultaneous measurements of the MW-PC and PL spectrum resolved transients. UV laser beam is properly attenuated by neutral filters and directed on the sample surface for excitation of MW-PC and PL signals. The MW-PC signal is collected from the excited area by microwave (MW) coaxial needle-tip antenna and registered by the MW detection system. The PL light is collected from a bisector between the incident and reflected UV laser beams and filtered (by UV 100\% mirror for excitation wavelength) and focused by quartz lens onto slit of the grating monochromator. The spectrally dispersed PL light transients are registered by photomultiplier. Both signals are simultaneously recorded at different channels of digital $1 \mathrm{GHz}$ band oscilloscope. (b) A sketch of the sample positioning in measurements.

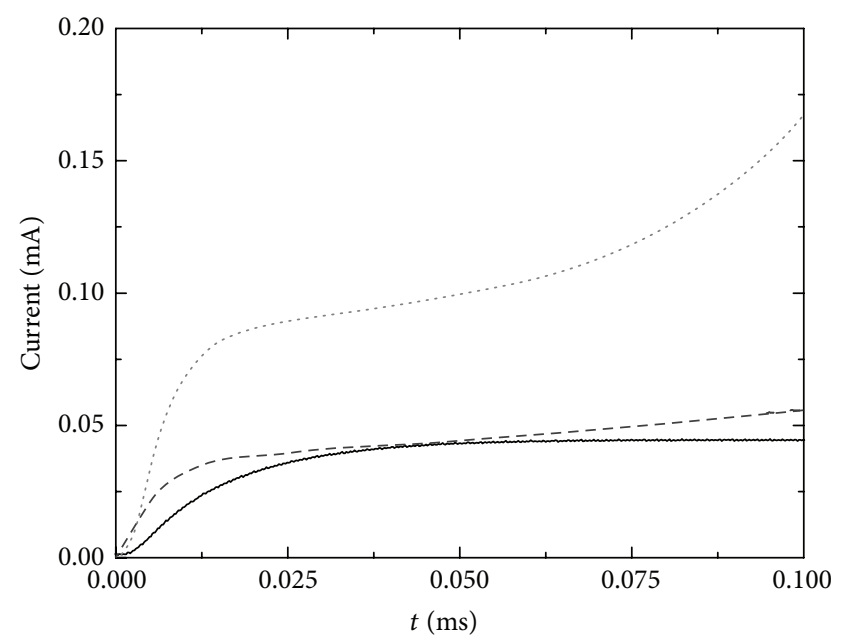

$\mathrm{CdS}-\mathrm{Cu}_{2} \mathrm{~S}$ sample of:
- I-type
$\ldots$. II-type
- - - III-type

Figure 2: The typical BELIV transients observed in different type $\mathrm{CdS}-\mathrm{Cu}_{2} \mathrm{~S}$ junction structures.

concentration of shallow traps. For the I-type samples, a geometrical capacitance $C_{g}$ can be only evaluated, which was found to be $C_{g}=3 \mathrm{nF}$, and this $C_{g}$ value is in agreement with that value calculated using the independently measured thickness $d$ and probed area $\mathrm{S}$ of CdS layer. The $\mathrm{Cu}_{1.96} \mathrm{~S}$, $\mathrm{Cu}_{1.92} \mathrm{~S}, \mathrm{Cu}_{1.81} \mathrm{~S}$, and other modifications together with $\mathrm{Cu}_{2} \mathrm{~S}$ were detected by XRD patterning. The richest XRD pattern of different phase precipitates was obtained in the samples of the I-type [18]. Additionally, the capacitance deep level transient spectroscopy technique modified by using optically induced conductivity regime (C-DLTS-OP) and photoionization spectroscopy (PIS) were applied to identify traps in CdS layer. These results are presented in detail elsewhere [19]. It was found that the I-type junction samples contain the richest structure of traps with different photoionization activation energies. The II-type and III-type samples exhibit two-three step structure of the measured PIS spectrum. The C-DLTSOP spectra can be recorded only in the samples of II- and III-type. There, only traps of the majority carriers have been distinguished within samples of the II- and III- types.

The junction area integrated characteristics of capacitance (BELIV), DLTS, and PIS characteristics on separate different types of samples are also dependent on spatial inhomogeneity of deposited layers. This inhomogeneity was confirmed by examination of the MW-PC transients. The inhomogeneity appears due to a system of distributed parameters, when nano-/microcrystalline volumes of rather perfect crystals are surrounded by disordered material. The dislocations and other extended imperfections located within these periphery areas, surrounding microcrystals, determine carrier trapping, recombination, and percolative transport. Excess carriers, brought into the periphery areas with space charge regions within Cottrell spheres of dislocations, are separated by space charge field, and their recombination is only mediated by diffusion, caused by excess carrier density gradients. Thereby, the aim of the present study was to identify a role of spatial inhomogeneity of CdS polycrystalline material by time and spectrally resolved carrier decay characteristics.

\subsection{Steady-State Photoluminescence Spectra and Confocal} Microscopy Images. The steady-state UV excitation induced photoluminescence spectra and microscopy images obtained on CdS samples of the I-type, the II-type, and the III-type are illustrated in Figures 3, 4, and 5, respectively. Two bands of photoluminescence have been observed within all types of samples, namely, a narrower band of green luminescence (G-PL) with a peak at $500 \mathrm{~nm}$ and a wider band of red luminescence (R-PL) with a peak at $700 \mathrm{~nm}$, Figures 3-5. 


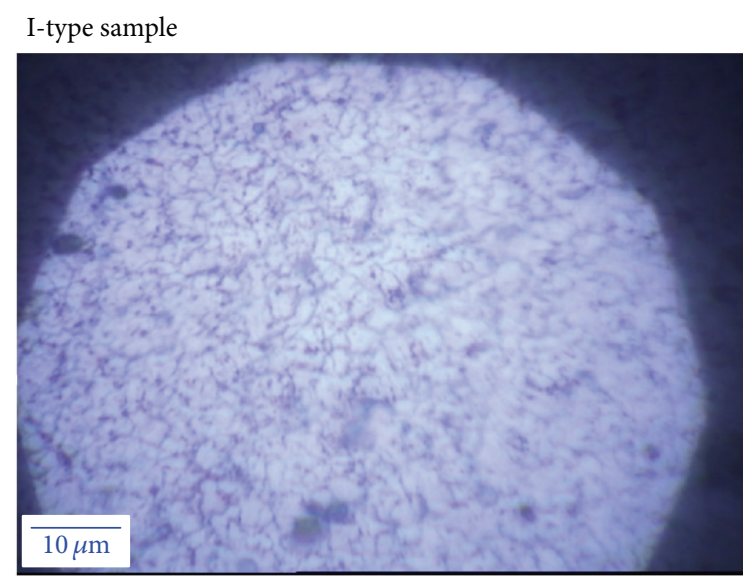

Optical image of scan area at 100x magnification

(a)

3000 CCD cts

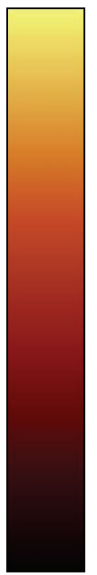

$0 \mathrm{CCD}$ cts

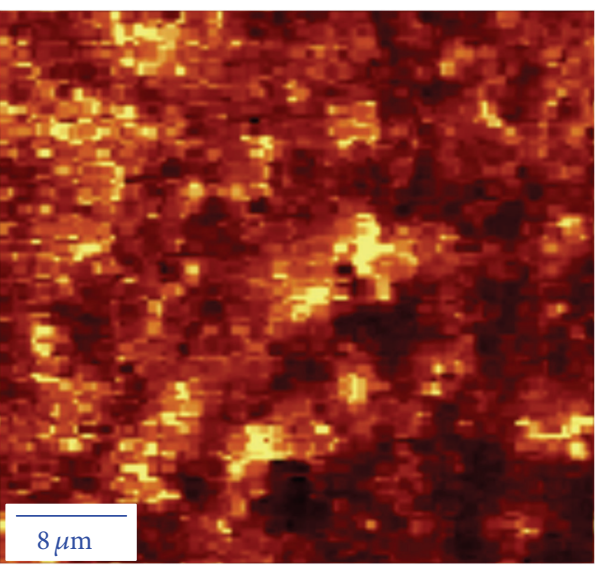

(c)

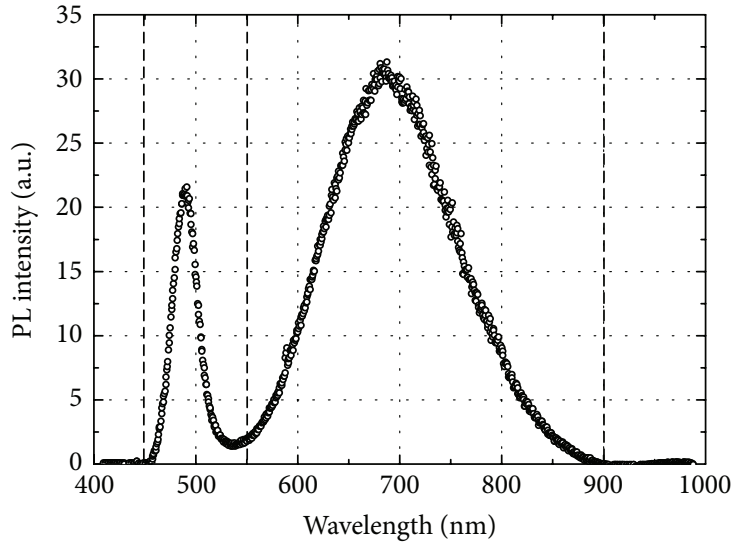

Spatially integrated PL spectrum

(b)

$6000 \mathrm{CCD}$ cts

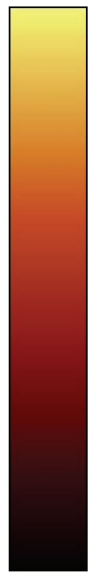

0 CCD cts

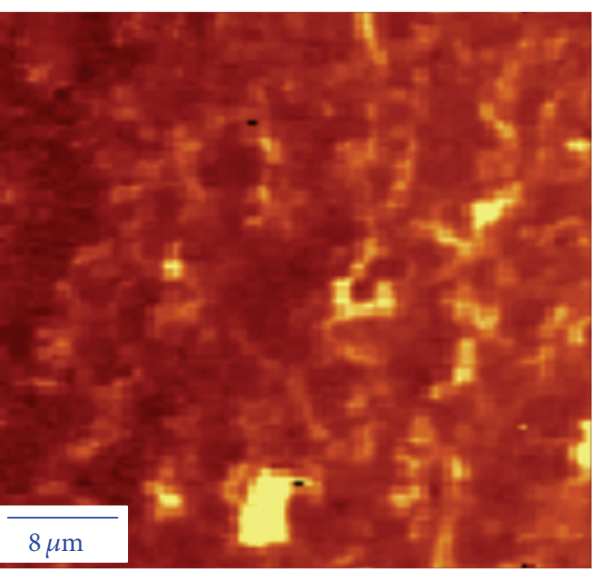

(d)

FIGURE 3: Optical field microscopy image (a), a PL spectrum integrated over inspected area (b), and the topography of the distribution of the green-light luminescence (c) and of the red-light luminescence (d), respectively, recorded for the CdS sample of the I-type.

It can be noticed, however, that the absolute values of PL intensity and a ratio between the green PL to the red PL intensities differ significantly, when comparing PL spectra for samples of these three types. The ratio $R_{\mathrm{PL}}=I_{\mathrm{G}-\mathrm{PL}} / I_{\mathrm{R}-\mathrm{PL}}$ of peak intensities obtained for the I-type CdS samples is close to unity, that is, $R_{\mathrm{PL}-\mathrm{I}-\mathrm{type}}<1$. These ratios are estimated to be $R_{\text {PL-II-type }} \geq 1$ and $R_{\text {PL-III-type }}>1.5$, respectively. Spatial distribution of the $I_{\mathrm{G}-\mathrm{PL}}$ and $I_{\mathrm{R}-\mathrm{PL}}$ was examined within microscopy (CMI) scan area by using spectral filters. It can be noticed within bottom pictures in Figures 3-5 that spatial distribution of the green PL (G-PL) and red PL (R-PL) radiative centres is obtained different among all three type samples; namely, centres of G-PL and R-PL are spatially separated for samples of the I- and III-type samples, while location of these G-PL and R-PL centres nearly coincides for samples of the II-type. This result confirms the enhanced generation current in BELIV transients registered for the samples of the II-type, indicating that deep traps are located in the same micro-crystallites.

Comparison of the confocal microscopy images and of the PL spectra among the CdS polycrystalline layers of different types implies that the most homogeneous CdS layer is obtained for the II-type samples. The smallest size $(\leq 1 \mu \mathrm{m})$ of the micro-crystallites is unveiled for the I-type samples, while the crystallites within layers of the II-type are the largest ones $(2-10 \mu \mathrm{m})$, as deduced from CMI. Location of the G-PL and R-PL centres is obtained to be the closest for the II-type layers (Figures 3-5). This indicates that traps responsible for G-R-PL donor-acceptor or band-deep level radiative recombination are most homogeneously distributed in the II-type samples. These observations are in agreement with BELIV characteristics, where the most qualitative CdS$\mathrm{Cu}_{2} \mathrm{~S}$ junctions (with clearly expressed barrier capacitance) were obtained on CdS substrates of the II-type. 


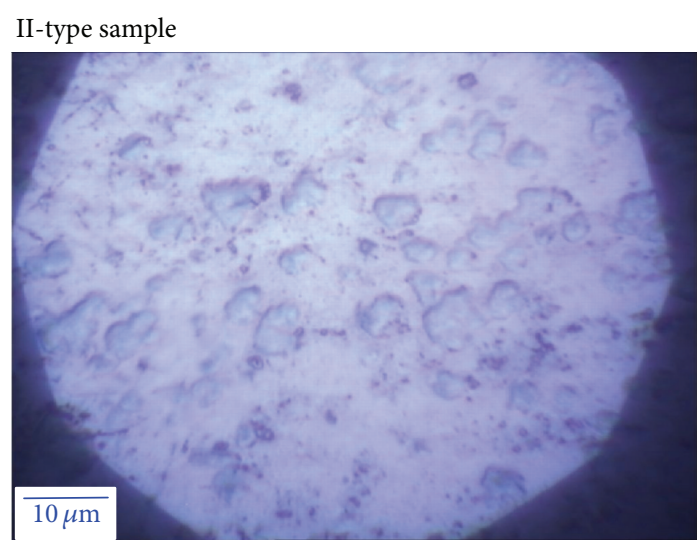

Optical image of scan area at 100x magnification

(a)
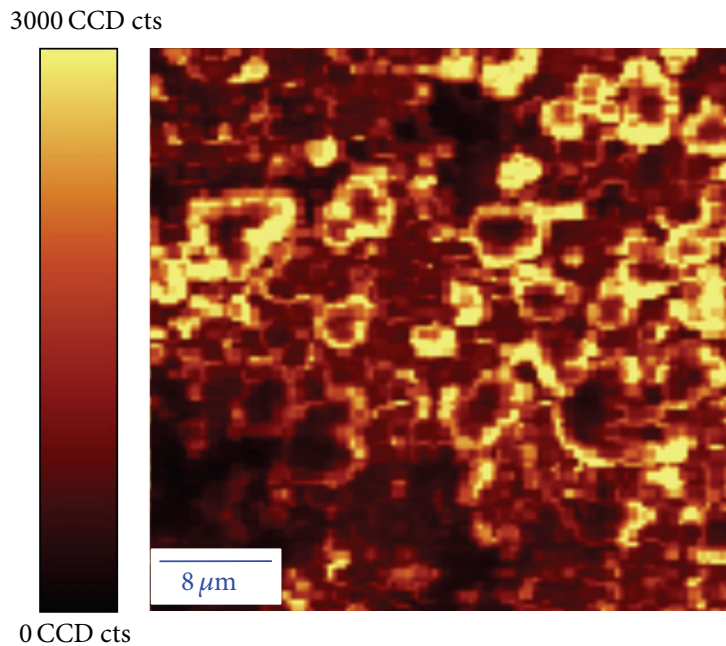

(c)

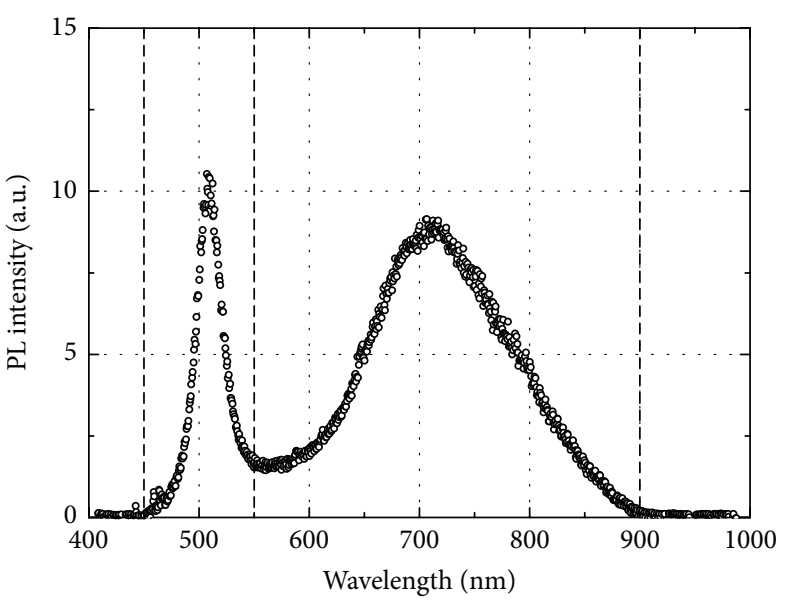

Spatially integrated PL spectrum

(b)

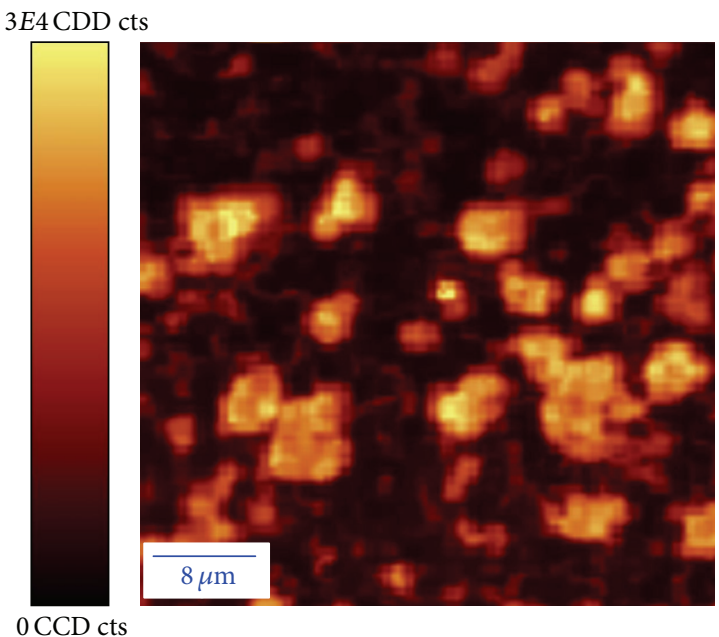

(d)

FIgURE 4: Optical field microscopy image (a), a PL spectrum integrated over inspected area (b), and the topography of the distribution of the green-light luminescence (c) and of the red-light luminescence (d), respectively, recorded on the CdS sample of the II-type.

\subsection{Characteristics of the Photoluminescence and Photoconductivity Transients}

4.3.1. Photoluminescence Quenching Effects. The simultaneously measured transients of photoluminescence (PL) and photoconductivity (MW-PC) under different background illumination are illustrated in Figure 6(a). The reversible luminescence photoquenching effect (PL-PQ) has been clearly observed, as a decrease of PL intensity with enhancement of the background illumination. In these experiments different bias illumination regimes, together with UV excitation of PL, have been implemented by carrying out measurements in dark, at room lighting and under illumination with incandescent lamp. The photoconductivity transients (Figure 6(a)), however, show nearly invariant excess carrier decay (MW-PC transient) shape. Two-componential MWPC transients with fast initial component and a longtail are inherent for all the investigated types of samples. Duration of the initial MW-PC transient component is in the same timescale (of several nanoseconds) with PL decay. However, the long-tail MW-PC relaxation, obtained by extrapolating this (MW-PC transient) curve to $t=0$ represents the nonexponential decay of excess carrier density due to nonradiative processes.

The photoluminescence photoquenching effect has been also resolved for the green CW UV light excited photoluminescence band (G-PL) by examination of the confocal microscopy images, highlighted by comparing PL intensity obtained without (Figure 6(b)) and with (Figure 6(c)) background illumination. The intensity of background illumination was varied using incandescent lamp. Comparison of GPL intensity of UV excited PL with and without background illumination is shown in Figure 6(d). The G-PL islands (Figure 6(b)), clearly observable within microscopy image without bias illumination, disappear (Figure 6(c)) when bias illumination is switched on. The changes of the amplitude of 


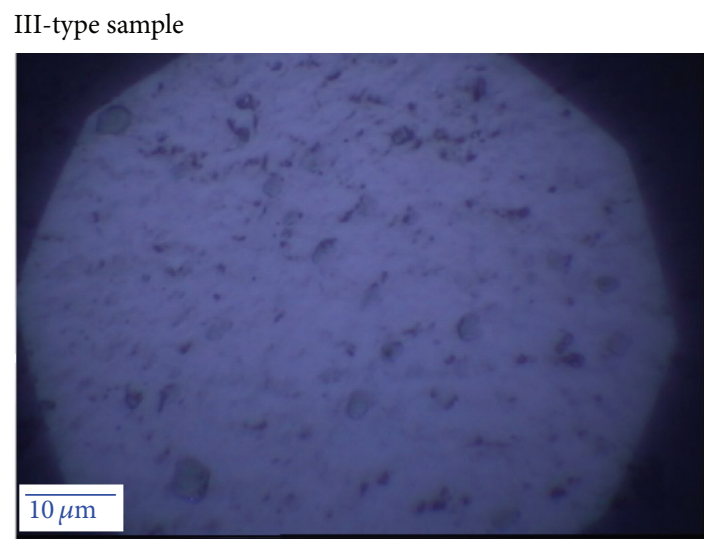

Optical image of scan area at 100x magnification

(a)

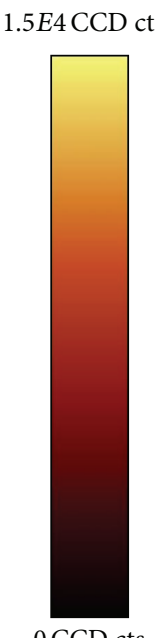

$0 \mathrm{CCD}$ cts

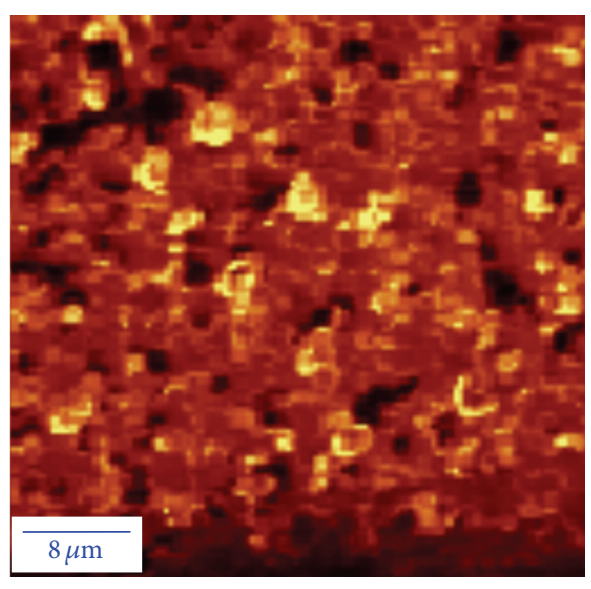

(c)

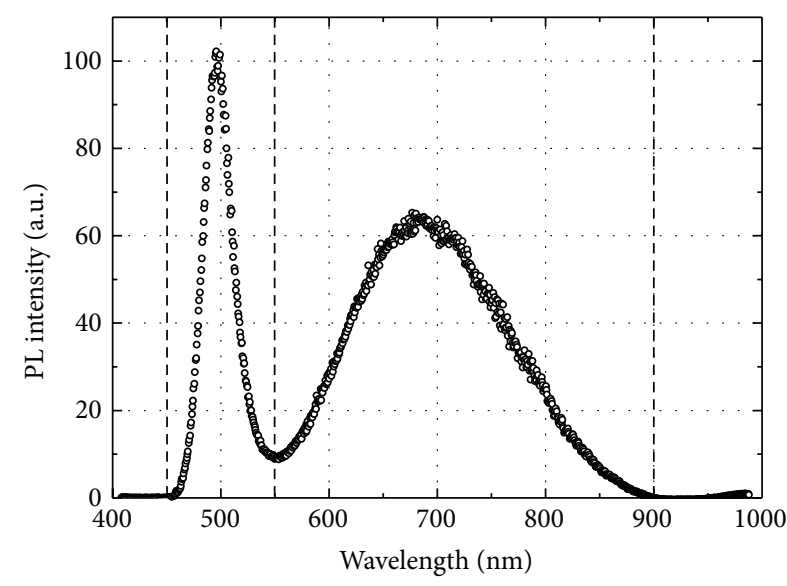

Spatially integrated PL spectrum

(b)

$3 E 4$ CDD cts

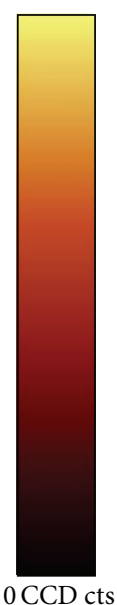

$0 \mathrm{CCD}$ cts

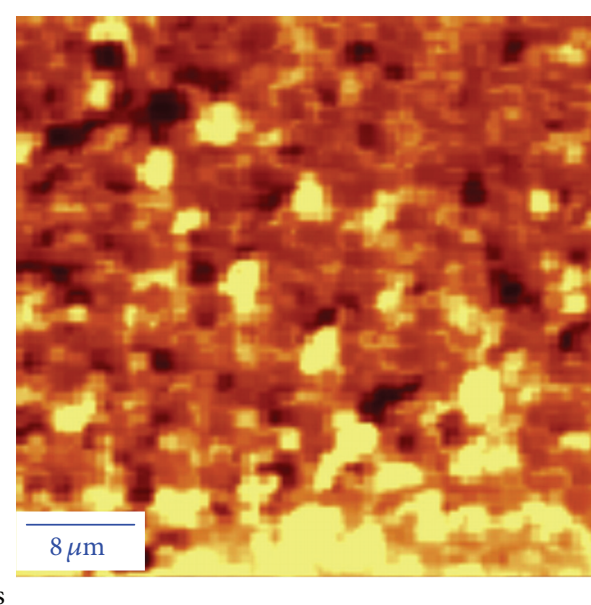

(d)

FIGURE 5: Optical field microscopy image (a), a PL spectrum integrated over inspected area (b), and the topography of the distribution of the green-light luminescence (c) and of the red-light luminescence (d), respectively, recorded on the CdS sample of the III-type.

PL peak are clearly seen for G-PL band, while intensity of the red PL band slightly increases within a long wavelength spectral wing. Recovery of a G-PL peak intensity occurs to be noticeably long at room temperature. Thus, the luminescence photoquenching effect seems to be accompanied with thermal quenching of PL. These luminescence photo- and thermal quenching effects are well-known in bulk crystals of CdS material [24] and are explained by interplay of several deep traps; those redistribute excess carrier flows among different radiative and nonradiative recombination centres within crystalline volumes. A sketch of redistribution of radiative recombination flows including potential barriers is presented in Figure 6(e). There, bias illumination modified (lowered) barrier can be a reason for G-PL quenching. The green photoluminescence can be ascribed to the nearly interband radiative recombination, assuming the band-gap value $E_{G}=2.42-2.47 \mathrm{eV}[25,26]$. Existence of the G-PL within microcrystalline volumes of the CdS polycrystalline material and PL-PQ effect imply a complicated interplay of defects in redistribution of the photopulse excited carriers within crystalline volumes of CdS.

These changes of the confocal microscopy and PL-PQ spectroscopy characteristics are more pronounced for the IItype samples. The G-PL can be associated with interband radiative transitions observed as PL peaked at $\sim 500 \mathrm{~nm}$. A reduction of the G-PL intensity due to PL-PQ determines a seeming shift of the R-PL peak (Figure 6(d)). Also, an increase of the steady-state R-PL intensity (within a long wavelength spectral wing) implies a switch of radiative electron-hole plasma [26] recombination flow (ascribed to G-PL) towards R-PL channel (associated with deep traps). On the basis of deep level parameters for these samples, resolved by the photoionization (PIS) and thermal-emission (DLTS) spectroscopy [19], the R-PL peaked at $700 \mathrm{~nm}$ can be ascribed to centres with activation energy $E_{\mathrm{A}, \mathrm{DL}}=1.75 \mathrm{eV}$. The latter $E_{\mathrm{A}, \mathrm{DL}}=1.75$ value is in good agreement with that 


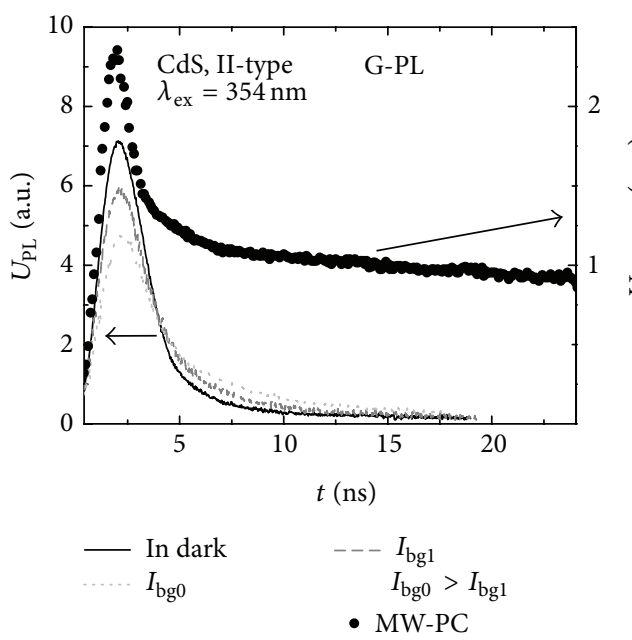

(a)

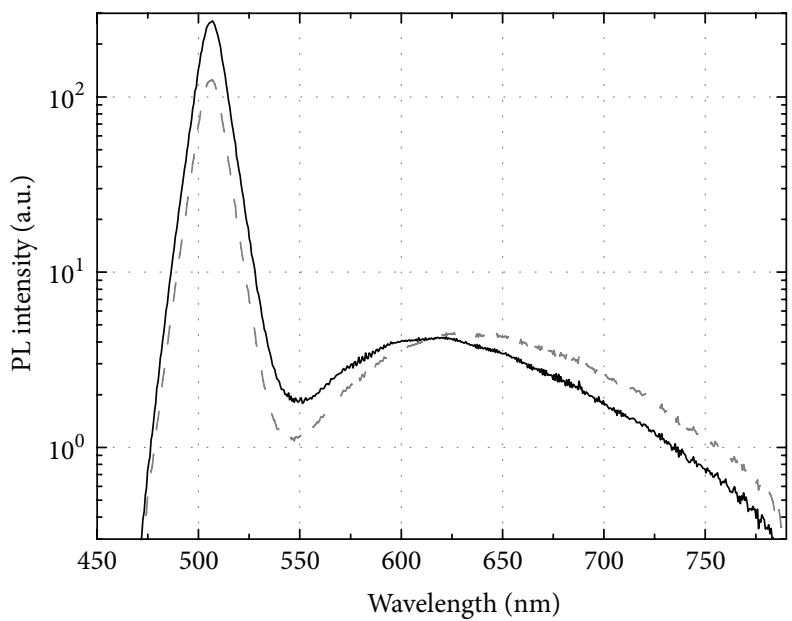

(d)
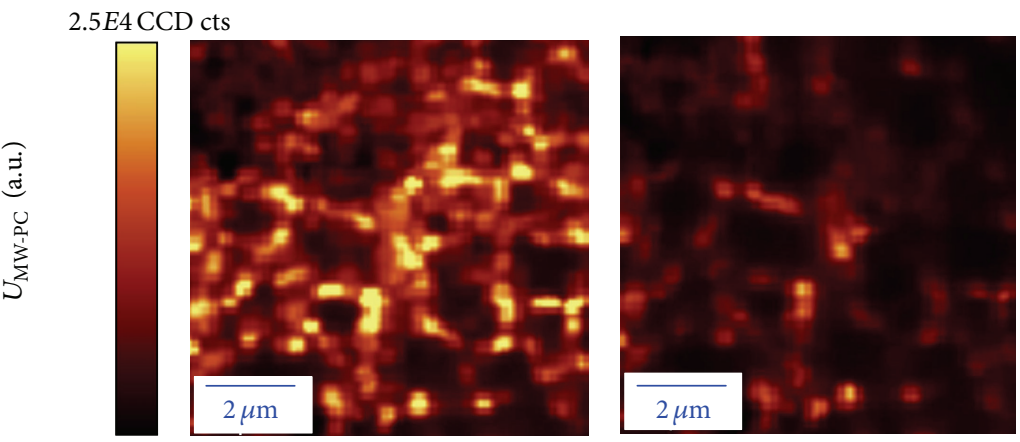

$1000 \mathrm{CCD}$ cts (b)

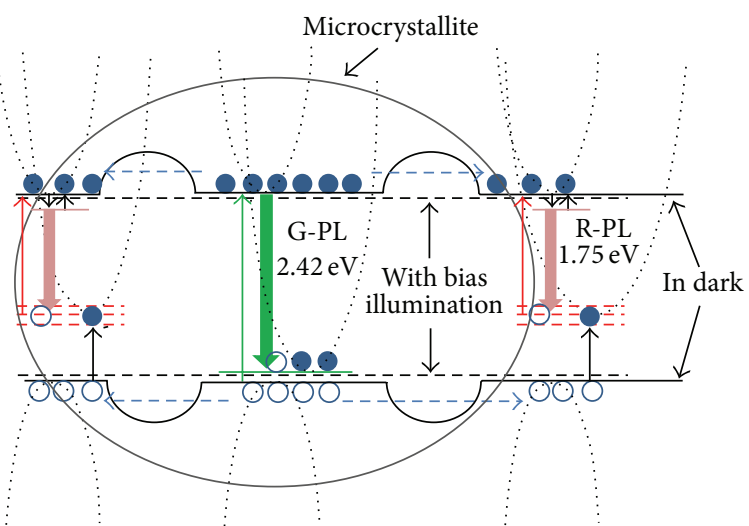

(e)

FIGURE 6: PL photoquenching characteristics: (a) comparison of MW-PC and PL transients varying intensity of background (bg) illumination. CdS surface PL intensity mapping in darkness (b), and under white light illumination (c), envisaged PL photoquenching effect, when PL integrated within spectral range from $450 \mathrm{~nm}$ to $750 \mathrm{~nm}$. (d) Spatially integrated PL spectrum for initial scan in darkness (black solid curve) and for scan made during background illumination (gray dash curve). (e) A sketch of photoluminescence quenching by redistribution of recombination flows under bias illumination.

$E_{\mathrm{DL}}=1.65 \pm 0.04 \mathrm{eV}[19]$ (extracted from photoionization spectra recorded on the same type CdS samples) including activation barriers within a configurational space [27].

\subsubsection{Photoluminescence and Photoconductivity Transients in} Different Type Samples. Complicated redistribution of excess carriers among the radiative and nonradiative recombination centres within polycrystalline $\mathrm{CdS}$ material is corroborated by examination of the MW-PC transients (Figure 7). The isomorphic MW-PC transients (within semilog plot) over wide range of time displays from nanosecond (top axis in Figure 7(a)) to millisecond (bottom axis in Figure 7(a)) scales have been observed. The invariant shape transients (seeming as two-componential) for different display time-scales imply entirely nonexponential carrier decay process, when MW-PC signal is integrated over excitation laser beam spot area.
Actually, the MW-PC transients represent a sum of excess carrier densities decay through both radiative (PL) and nonradiative $\left(n_{\text {ex,as }}\right)$ recombination, averaged over excitation spot area. The asymptotic decay component $\left(n_{\text {ex,as }}\right)$ can be rather precisely simulated using stretched exponent model $n_{\text {ex,as }}(t)=n_{\text {ex,as }}(t=0) \exp \left[-\left(t / \tau_{\text {se }}\right)^{\alpha}\right]$, with fractional index $\alpha$ and specific time-constant $\tau_{\text {se }}$ parameters, when a peak amplitude is normalized to unity $\left(\left.n_{\text {ex,as }}(t)\right|_{\text {normalized }}=\right.$ $\left.\exp \left[-\left(t / \tau_{\text {se }}\right)^{\alpha}\right]\right)$, Figure $7(\mathrm{~b})$. Extrapolating the fitted $n_{\text {ex,as }}(t)$ to the initial instant $(t=0$, ascribed to the MW-PC initial peak) of the experimental MW-PC transient $\left(U_{\mathrm{MW}-\mathrm{PC}}(t)\right)$, a $n_{\text {ex,PL }}(t)$ density of excess carriers decaying by radiative transitions (as a differential between the initial and asymptotic components) can be evaluated. The simulated ratio $n_{\text {ex }, \text { PL }}(t) / n_{\text {ex }, \text { PL }}(0)=\left\{U_{\text {MW-PC }}(t) / U_{\text {MW-PC }}(0)\right\}-\exp \left[-\left(t / \tau_{\text {se }}\right)^{\alpha}\right]$ is compared with experimental PL transient within an inset 


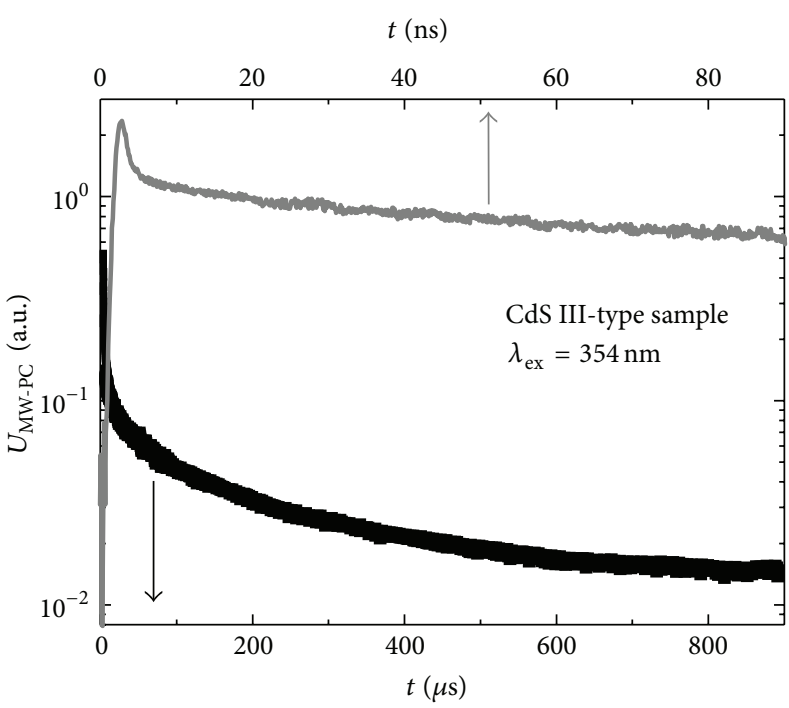

(a)

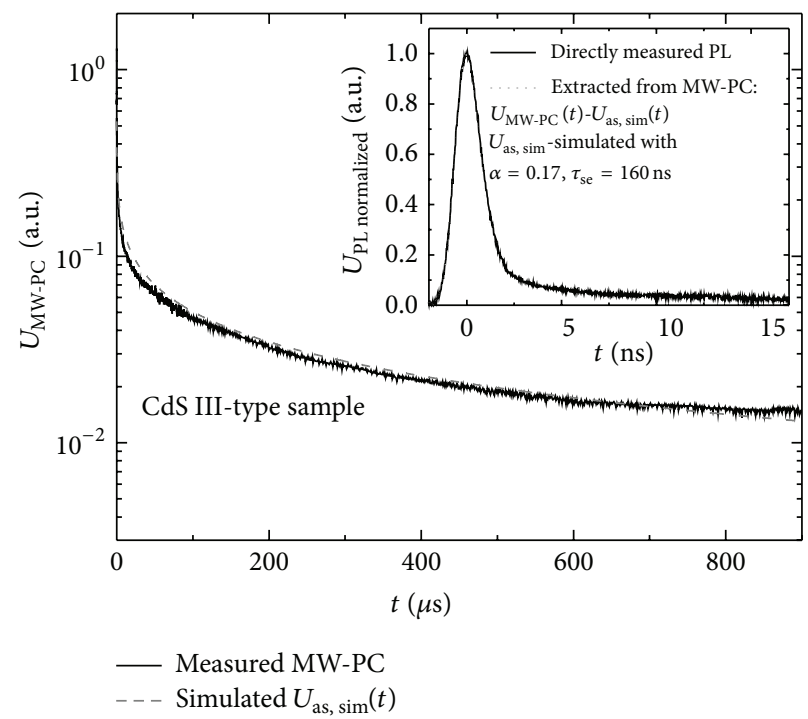

(b)

Figure 7: (a) MW-PC transients, recorded using different display scales (top-ns scale, grey curve; bottom- $\mu$ s scale, black curve) and represented within semilog plot. (b) Comparison of simulated components of PL (in the inset, by subtracting the fitted and simulated stretched exponent from the experimental decay curve) and MW-PC (simulated stretched exponent relaxation) with experimental ones.

of Figure 7(b). It can be deduced from Figure 7(b) that the simulated main components of the MW-PC transient, shown in Figure 7(a), fit well the experimental MW-PC and PL transients, simultaneously measured on the same sample.

A comparison of MW-PC and PL transients obtained in different type CdS samples is presented in Figure 8. It can be noticed that the initial carrier decay rate through the nonradiative recombination channels, highlighted within MW-PC transients, is faster in samples of I-type containing the lowest equilibrium carrier density. While an amplitude of the asymptotic relaxation for I-type sample is larger than that for II- and III-type samples. The observed differences of MW-PC transients can be explained by larger volumes of microcrystals in II- and III-type samples. Owing to the larger volumes (and ratio of bulk material to crystallite surface) of microcrystals in II- and III-type samples, as observed in confocal microscopy images, Figures 3-5, the radiative (G-PL) interband recombination efficiency is elevated in IIand III-type samples. The radiative interband recombination seems to be responsible for the fast initial decay component.

Excess carrier decay at microcrystal boundaries and within disordered structure surrounding the microcrystals appears through carrier capture limited random-walk. The latter process leads to the stretched exponent inherent behaviour of carrier density relaxation. The rate of asymptotic carrier density changes (Figure 8(b)) depends on the structure of disordered material, which is characterized by the fractional index $\alpha$ and by a specific stretched exponent lifetime $\tau_{\text {se }}$. The PL transients also exhibit different initial (Figure $8(\mathrm{c})$ ) and asymptotic (Figure $8(\mathrm{~d})$ ) PL relaxation.
The slowest initial PL decay is again observed in I-type samples. While asymptotic PL relaxation is inherent for all the investigated types of $\mathrm{CdS}$ samples, but rate of carrier decay within this asymptotic relaxation component is different for various types of samples. These observations imply that processes of radiative recombination, during rather long time of about 200 ns (Figure 8(d)), contemporize also in microcrystals surrounding structure by exchange of carriers between microcrystals and their boundary area. The asymptotic tail of PL transients (Figure 8(d)) can be also associated with PL spectrum changes under varied excitation density (Figure 9). The G-PL photoquenching is clearly expressed for all types of samples. A shift of G-PL peak intensity to the long wavelength (R-PL) wing peak intensity appears also with increase of pulsed excitation density (Figure 9).

This can be explained by excess carrier density accelerated radiative recombination through trap levels, assuming a nonradiative capture of carriers before radiative transition. Here, a barrier inherent behaviour of carrier transport mediated radiative recombination can also be implied. It can be assumed that barrier widens and sinks with increase of excess carrier density, and thereby energy of emitted photons decreases. Such a behaviour can be explained by barrier existence for different recombination counter-partners, that is, holes and electrons involved [26]. On the other hand, these PL spectral shifts, accompanied by PL photoquenching, can be alternatively explained through processes governed by interplay of point traps [24]. Here, the switch of recombination flow from deeper trap to shallower one (being, $r$ and $s$ centres in nomenclature of $[24,28])$ is the main reason of spectral changes. 

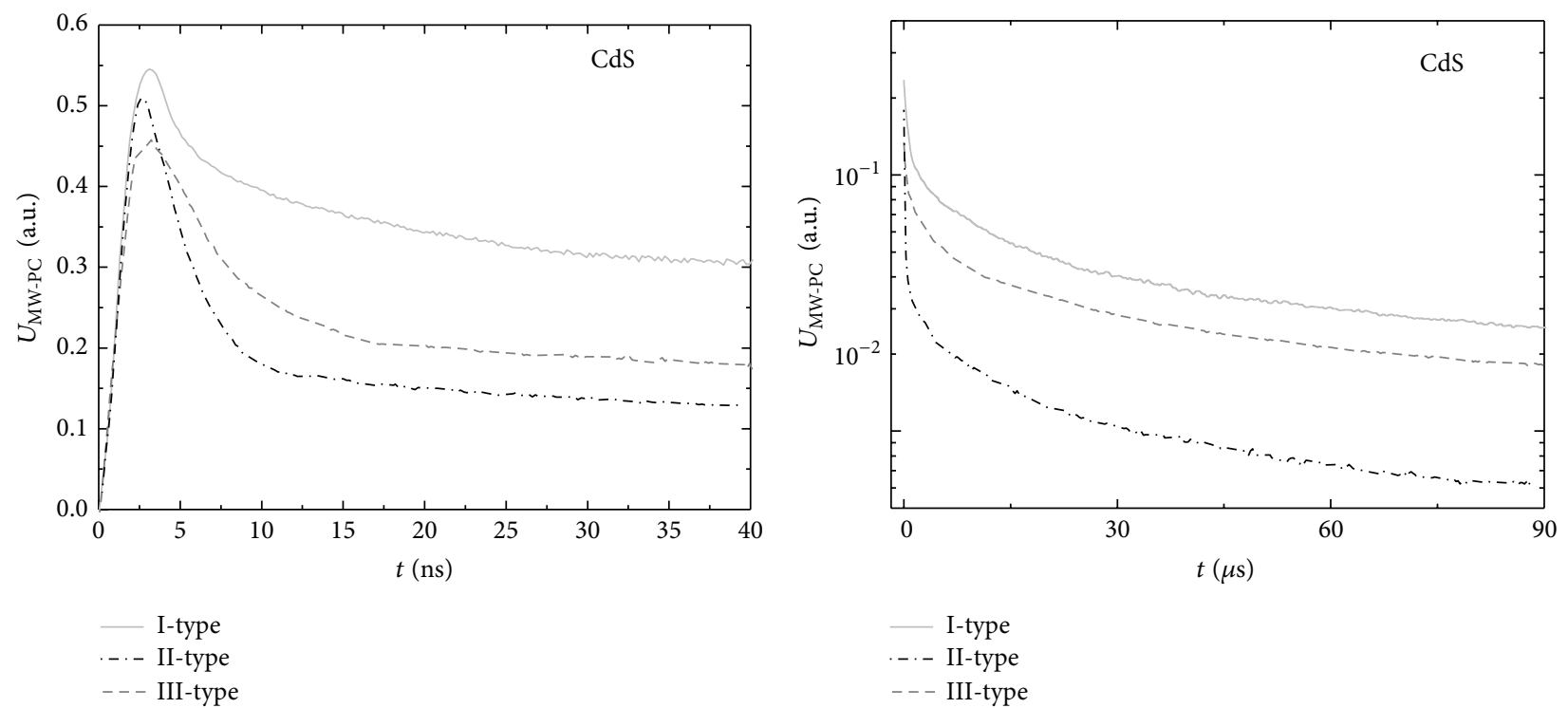

(a)

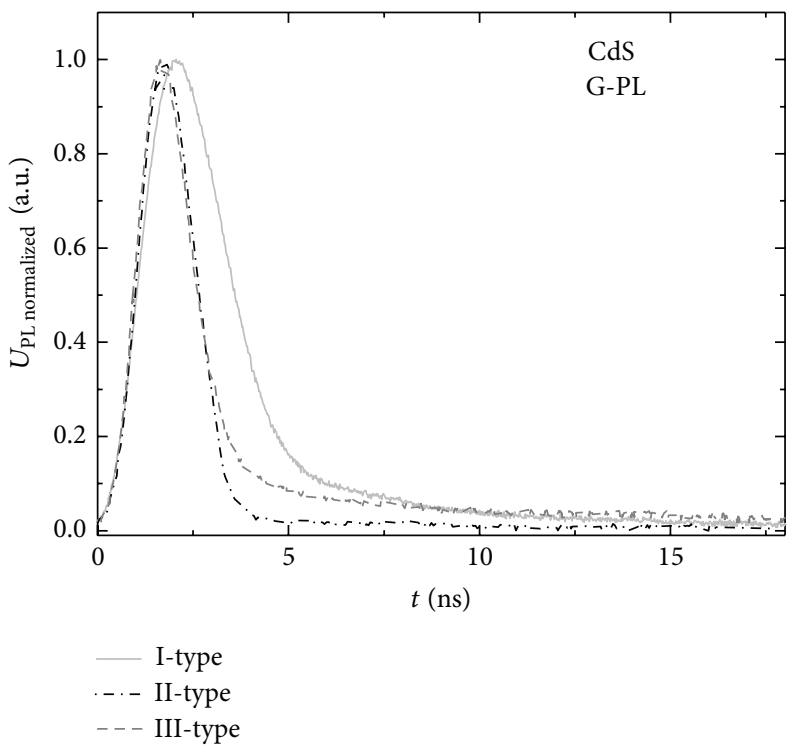

(b)

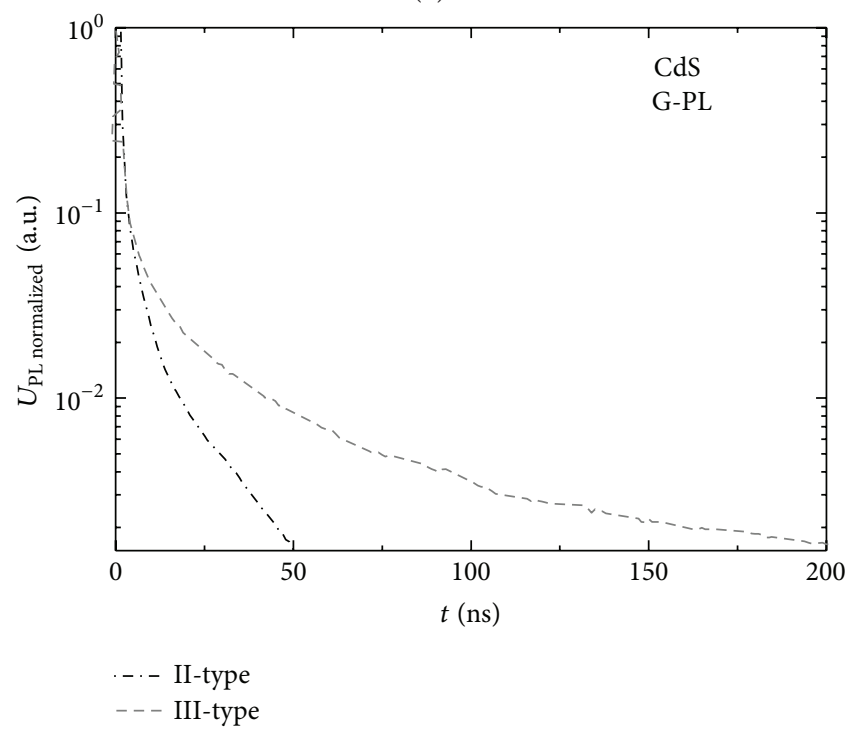

(c)

(d)

FIGURE 8: Comparison of the MW-PC initial (a) and asymptotic (b) decays correlated with G-PL of the initial (c) and asymptotic (d) relaxation components in polycrystalline layers of I-, II-, and III-type samples of CdS material.

Dependence of the asymptotic PL component on sample type is confirmed by the MW-PC transients recorded for different type samples (Figure 10). This dependence can be roughly estimated (Figure 10(a)) by analysis of the ratio of the amplitudes $r_{\text {asti }}=U_{\text {as }} / U_{\text {in }}$, using values of the asymptotic $U_{\text {as }}$ relaxation, extrapolated to the initial decay moment $(t=0)$, and that of the initial decay peak $U_{\text {in }}$. The smallest $r_{\text {asti }}$ ratio has been obtained for II-type samples, while this ratio $r_{\text {asti }}$ is the largest for the I-type samples. The large relative amplitude $U_{\text {as }}$ indicates the enhanced role of the disorder, where excess carrier nonradiative recombination is governed by carrier random-walk transport. The smaller $r_{\text {asti }}$ is, the most efficient integral PL efficiency is obtained. There, carrier recombination within micro-crystallites prevails within initial component of MW-PC transient.

4.3.3. Stretched Exponential Relaxation of Photoconductivity. A linearization of the $U_{\text {as }}$ within MW-PC transients is possible when using a stretched exponent approximation; for example, $[29,30] n_{\mathrm{ex}}(t)=n_{\mathrm{ex}}(t=0) \exp \left[-\left(t / \tau_{\mathrm{se}}\right)^{\alpha}\right]$, with fractional index $\alpha$. Such a display of a MW-PC transient is illustrated in Figure 10(b). Fractional index $\alpha$ is obtained from a linear fit of $U_{\text {as }}(t)$ variations using a plot of the double logarithm for normalized $U_{\text {as }}$ values versus 


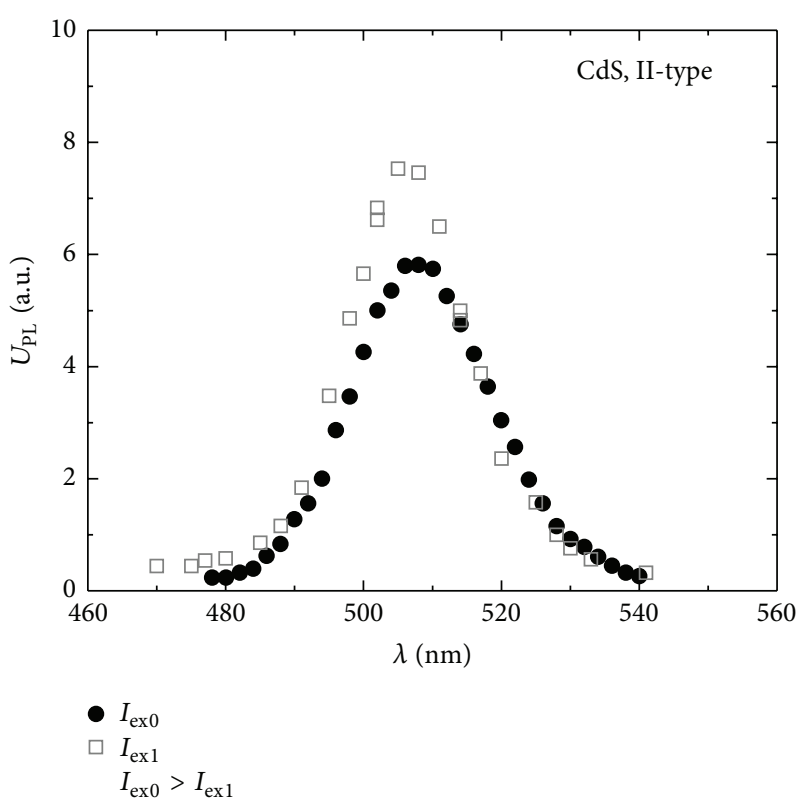

FIGURE 9: Comparison of PL spectra under pulsed excitation of varied intensity in II-type sample. The G-PL peak intensity decreases and peak slightly shifts to the long wavelength wing with increase of pulsed UV excitation intensity: $I_{\mathrm{ex} 0}$ (solid symbols) > $I_{\text {exl }}$ (hollow symbols).

a logarithm of time, that is, $\ln \left(\ln \left(U_{\mathrm{MWR}}(0) / U_{\mathrm{MWR}}(t)\right)\right)$ versus $\ln (t)$. Values of $\alpha$ can then be associated with material disorder characteristics, such as scaling exponents $d_{w}, d_{f}, \zeta$ $[31,32]$. Additionally a fractal factor $f$, defined as a measure of compression of configuration space, can be employed in analysis of the fractional index $\alpha$. These parameters had been introduced within axiomatic Scher-Lax-Phillips (SLP) model $[33,34]$ which provides an unambiguous way to interpret experimental results. This approach has been exploited in analysis of the microwave probed photoconductivity $U_{\text {as }}$ in different types of CdS polycrystalline layers in this work.

The SLP model provides a straightforward path to analyze the experimental values of the fractional index $\alpha$. This model is built upon the assumptions that the dissipation of excitations occurs via a system of fixed randomly distributed traps, while the system is microscopically homogenous. From here it follows that in the asymptotic limit the system exhibits SER with the fractional index defined as $\alpha=d /(d+2)$, where $d$ denotes the dimensionality. Then, it is supposed that in some cases the relaxation dynamics is governed by shortand long-range interactions, which lead to a compression of configuration space and, consequently, to a more general definition of $\alpha$; that is, $\alpha=d^{*} /\left(d^{*}+2\right)$, where $d^{*}=f d$ is the effective dimensionality. In a wide variety of glasses, $f$ is usually equal to 1 or $1 / 2$, being specific values to shortrange and mixed short- and long-range forces, respectively. However, for polycrystalline materials $f$ can attain even smaller values [34], since a large part of the effective degrees of freedom is inactive due to clustering. Values of fractional index $\alpha$ and fractal factor $f$ for samples of various types, obtained from curves depicted in Figure 10(b), are presented
TABLE 1: Parameters of disorder in samples of different type.

\begin{tabular}{lccc}
\hline Parameter & \multicolumn{3}{c}{$\begin{array}{c}\text { Sample type } \\
\text { II-type }\end{array}$} \\
\hline$\alpha$ & 0.20 & 0.16 & III-type \\
$f$ & 0.17 & 0.13 & 0.17 \\
\hline
\end{tabular}

in Table 1. From these results, it can be inferred that samples of the II-type and the III-type are structurally similar, and that samples of the I-type possess a distinguishably disordered structure.

4.4. Discussion. The performed study of the CdS polycrystalline material layers by applying combination of different techniques enabled us to separate a few types of samples those differ in density of doping and carrier capture centres. The parameters of the CdS substrate (base) layer and the heterojunction extracted by using different techniques are summarized in Table 2. The samples had been sorted into three types according to differences in BELIV transient shapes (Figure 2), which were identified assuming variation of relative densities of dopants and carrier capture, caused by slight changes of layer deposition regimes. The dark resistivity is very large in samples of the I-type due to a lack of freecarriers; thus, junction appeared to be fully depleted without external applied voltage. The enhanced dark resistance can be explained by the large density of traps which considerably exceeds the density of dopants. Value of free-carrier density of the $n_{0}=1.5 \times 10^{13} \mathrm{~cm}^{-3}$ is estimated for junctions of II- and III-type, and it determines concentration of shallow traps.

Spatial inhomogeneity, caused by dislocations and other extended imperfections located within these periphery areas surrounding microcrystals, determines carrier recombination and transport features. Excess carriers, brought to the periphery areas containing space charge regions, are separated by space charge field, and their recombination is only mediated by diffusion, owing to excess carrier density gradients. Carrier capture within local space-charge region fields seems to be potential barrier modified. Screening of this barrier is dependent on equilibrium and excess carrier density. Thereby, the observed G-PL peak intensity in different type samples and its spectral shifts can be explained by variation of carrier densities in various type polycrystalline structures.

Different (fast and slow) carrier decay components should be ascribed to different microareas of CdS polycrystalline material. It has been confirmed by photoluminescence (PL) microscopy that PL signal contains a granular structure. Thus, the simultaneous control of photoconductivity (ascribed to integrated over excitation beam spot area) and green photoluminescence (associated with interband recombination within micro-crystalline volumes) spectrum and time resolved signals can be a tool to resolve an impact of different microvolumes to carrier decay parameters.

Two spectral bands of photoluminescence have been observed within all types of samples: a narrower band of 


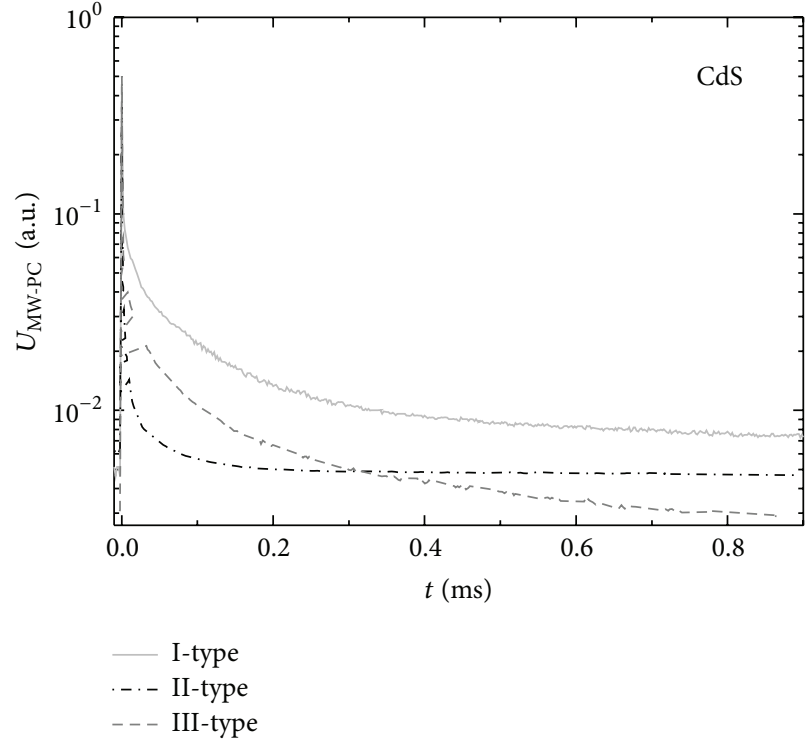

(a)

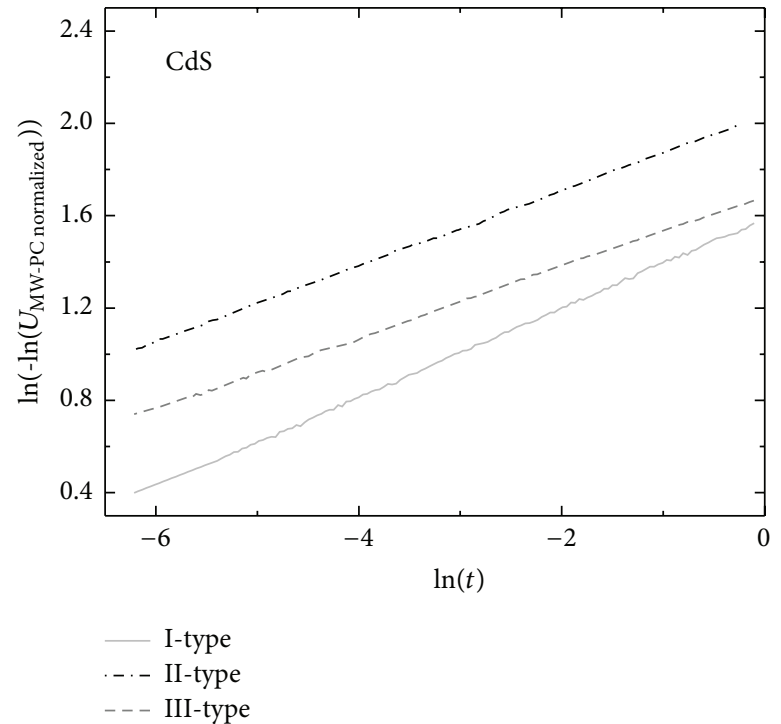

(b)

FIGURE 10: Comparison of the MW-PC transients recorded in I- to III-types of CdS samples at $354 \mathrm{~nm}$ wavelength pulsed excitation (a), and display of transients in the stretched exponent scale (b).

green luminescence peaked at $500 \mathrm{~nm}$, which can be associated with interband radiative recombination and a wider spectral band of red luminescence with a peak at $700 \mathrm{~nm}$, which can be ascribed to deep trap mediated radiative transitions. The ratio between the green PL to the red PL intensities, as $R_{\mathrm{PL}}=I_{\mathrm{G}-\mathrm{PL}} / I_{\mathrm{R}-\mathrm{PL}}$, is found to be $R_{\mathrm{PL}-\mathrm{I} \text {-type }}<$ 1 for the I-type CdS samples, while values of this ratio are estimated to be $R_{\mathrm{PL}-\text { II-type }} \geq 1$ and $R_{\mathrm{PL}-\mathrm{III}-\mathrm{type}}>1.5$, respectively. Centres of G-PL and R-PL are spatially separated for the I- and III-type samples, while value $R_{\text {PL-III-type }}>$ 1.5 indicates the smallest density of deep traps in III-type samples. Spatial location of these G-PL and R-PL centres nearly coincides with samples of the II-type, as identified from images in Figures 3-5.

The confocal microscopy images among the CdS polycrystalline layers of different types imply that the most homogeneous CdS layer is obtained for the II-type samples, deposited at $260^{\circ} \mathrm{C}$ for $75 \mathrm{~min}$. While the PL spectra show that interband recombination within microcrystals (associated with G-PL) prevails in III-type samples, deposited at $260^{\circ} \mathrm{C}$ for $80 \mathrm{~min}$. This is in agreement with BELIV characteristics, where the most qualitative $\mathrm{CdS}-\mathrm{Cu}_{2} \mathrm{~S}$ junctions (with clearly expressed barrier capacitance and a relatively small leakage current) were obtained on CdS substrates of the III-type.

The photoluminescence photoquenching effect has been clearly observed (Figures 6 and 9). The photoluminescence photoquenching effect has been ascribed to the microcrystalline areas within polycrystalline $\mathrm{CdS}$ material and resolved for the green photoluminescence band (G-PL) by examination of the confocal microscopy images without and with background illumination, using incandescent lamp. Existence of the G-PL within microcrystalline volumes of the CdS polycrystalline material and PL-PQ effect imply a complicated interplay of defects in redistribution of the photopulse excited carriers over crystallites. The PL-PQ effect is more pronounced for the II-type samples. This can be explained by competition and interplay of radiative and nonradiative recombination centres in redistribution of carrier flows, located within the same crystalline volumes. For all the investigated types of samples, a shift of PL peak appears with decrease of excitation density (Figure 9). This implies a barrier specific behaviour of carrier transport to radiative recombination centres.

Two-componential MW-PC transients with fast initial component and a nonexponential long tail are inherent for all the investigated types of samples, and this is a feature of the polycrystalline structure. Duration of the initial MWPC transient component is in the same time-scale (of several nanoseconds) with G-PL decay; thus, it represents carrier density relaxation within crystalline volumes. Only rather small part of excess carriers is shared within radiative recombination processes, observable in PL signal changes. The radiative recombination processes represent the excess carrier density changes within microcrystals, and thereby the time-scale of the initial MW-PC ( $U_{\text {in }}$ as a sum of PL and nonradiative recombination, incorporated within $U_{\text {as }}$ ) and of G-PL signals coincide. The MW-PC response amplitude is proportional to the whole density of excess carriers. Thus, ratio of amplitudes $r_{\text {asti }}=U_{\text {as }} / U_{\text {in }}$ of the MW-PC relaxation components can be a measure for carrier densities decaying within microcrystals and surrounding volume of CdS polycrystals investigated (Figures 7, 9, and 10). The observed differences of MW-PC transients, when comparing different type samples, can be explained by largest volumes (proven by CMI) of microcrystals in the II-type samples. The excess carriers decay within microcrystals through radiative 
TABLE 2: Parameters of different type CdS samples.

\begin{tabular}{|c|c|c|c|}
\hline \multirow{2}{*}{ Parameter } & \multicolumn{3}{|c|}{ Sample type } \\
\hline & I-type & II-type & III-type \\
\hline Technological regime & $\begin{array}{l}T_{\mathrm{dep}}=220^{\circ} \mathrm{C} \\
t_{\mathrm{dep}}=60 \mathrm{~min}\end{array}$ & $\begin{array}{l}T_{\mathrm{dep}}=260^{\circ} \mathrm{C} \\
t_{\mathrm{dep}}=75 \mathrm{~min}\end{array}$ & $\begin{array}{l}T_{\mathrm{dep}}=260^{\circ} \mathrm{C} \\
t_{\mathrm{dep}}=80 \mathrm{~min}\end{array}$ \\
\hline Equilibrium density of carriers (CdS base) & $n_{0}<<10^{13} \mathrm{~cm}^{-3}$ & $n_{0} \geq 1.5 \times 10^{13} \mathrm{~cm}^{-3}$ & $n_{0} \geq 1.5 \times 10^{13} \mathrm{~cm}^{-3}$ \\
\hline $\begin{array}{l}\mathrm{Cu}_{2} \mathrm{~S}-\mathrm{CdS} \text { heterostructure } \\
\text { (pulsed capacitance-BELIV) }\end{array}$ & $\begin{array}{l}\text { Capacitor-like [18], } \\
\text { insulator-specific state } \\
\text { of CdS base material }\end{array}$ & $\begin{array}{l}\text { Junction-like [18], } \\
\text { generation current } \\
\text { component in CdS } \\
\text { base is pronounced }\end{array}$ & $\begin{array}{l}\text { Junction-like [18], } \\
\text { barrier charging } \\
\text { current prevails }\end{array}$ \\
\hline $\begin{array}{l}\text { Activation energy of the dominant traps } \\
\text { (DLTS, PIS) }\end{array}$ & $\begin{array}{c}\mathrm{n} / \mathrm{a} \\
\Delta E_{\mathrm{PIS}}[19] \\
1.17 \pm 0.02 \mathrm{eV} \\
1.65 \pm 0.02 \mathrm{eV} \\
2.19 \pm 0.04 \mathrm{eV}\end{array}$ & $\begin{array}{c}\Delta E_{\text {DSLTS }}[19] \\
0.22 \pm 0.02 \mathrm{eV} \\
0.40 \pm 0.02 \mathrm{eV} \\
\Delta E_{\text {PIS }}[19] \\
1.21 \pm 0.02 \mathrm{eV} \\
1.56 \pm 0.02 \mathrm{eV} \\
2.19 \pm 0.04 \mathrm{eV}\end{array}$ & $\begin{array}{c}\Delta E_{\text {DSLTS }}[19] \\
0.22 \pm 0.02 \mathrm{eV} \\
0.31 \pm 0.02 \mathrm{eV} \\
\Delta E_{\text {PIS }}[19] \\
1.19 \pm 0.02 \mathrm{eV} \\
1.62 \pm 0.02 \mathrm{eV} \\
2.19 \pm 0.04 \mathrm{eV}\end{array}$ \\
\hline $\begin{array}{l}\text { Grain size/homogeneity of distribution } \\
\text { crystal grains in CdS substrate (MCI) }\end{array}$ & $\begin{array}{l}\quad<1 \mu \mathrm{m} \\
\text { inhomogeneous }\end{array}$ & $\begin{array}{c}2-10 \mu \mathrm{m} \\
\text { homogeneous }\end{array}$ & $\begin{array}{c}2-10 \mu \mathrm{m} \\
\text { inhomogeneous }\end{array}$ \\
\hline $\begin{array}{l}\text { Instantaneous lifetime of the initial decay } \\
\text { (MW-PC) }\end{array}$ & $3 \mathrm{~ns}$ & $5 \mathrm{~ns}$ & $10 \mathrm{~ns}$ \\
\hline $\begin{array}{l}\text { Instantaneous lifetime of the asymptotic } \\
\text { decay (MW-PC) }\end{array}$ & $>100 \mu \mathrm{s}$ & $>800 \mu \mathrm{s}$ & $>400 \mu \mathrm{s}$ \\
\hline $\begin{array}{l}\text { Ratio of the amplitudes of the asymptotic to } \\
\text { the initial MW-PC decay } r_{\text {asti }}=U_{\text {as }} / U_{\text {in } t=0}\end{array}$ & 0.25 & 0.01 & 0.04 \\
\hline $\begin{array}{l}\text { Instantaneous lifetime of the initial PL decay } \\
\text { G-PL peaked at } 500 \mathrm{~nm} \text { (TR-PL) }\end{array}$ & $\begin{array}{c}5 \mathrm{~ns} \\
\text { PL photo-quenching } \\
\text { observable }\end{array}$ & $\begin{array}{l}2 \mathrm{~ns} \\
\text { the strongest } \mathrm{PL} \\
\text { photo-quenching }\end{array}$ & $\begin{array}{c}2 \mathrm{~ns} \\
\text { strong PL } \\
\text { photo-quenching }\end{array}$ \\
\hline $\begin{array}{l}\text { Instantaneous lifetime of the asymptotic } \\
\text { decay for R-PL peaked at } 700 \mathrm{~nm} \text { (TR-PL) }\end{array}$ & $\mathrm{n} / \mathrm{a}$ & $40 \mathrm{~ns}$ & $150 \mathrm{~ns}$ \\
\hline $\begin{array}{l}\text { Ratio of the G-PL \& R-PL intensities } \\
R_{\mathrm{PL}}=I_{\mathrm{G}-\mathrm{PL}} / I_{\mathrm{R}-\mathrm{PL}} \text { (MCI-PLS) }\end{array}$ & $<1$ & $\geq 1$ & $\geq 1.5$ \\
\hline Fractional SER index $\alpha$ (MW-PC) & 0.20 & 0.16 & 0.17 \\
\hline Fractal factor $f$ (SER) & 0.17 & 0.13 & 0.14 \\
\hline
\end{tabular}

recombination, is associated with fast initial MW-PC and $\mathrm{PL}$ components. The slower $\mathrm{PL}$ and $\mathrm{MW}-\mathrm{PC}$ relaxation components can be ascribed to carrier recombination within microcrystals and their diffusion to microcrystal boundary.

A linearization of MW-PC transients is possible when using a stretched exponent approximation, $n_{\mathrm{ex}, \mathrm{as}}(t)=$ $n_{\text {ex,as }}(t=0) \exp \left[-\left(t / \tau_{\text {se }}\right)^{\alpha}\right]$, with fractional index $\alpha$ different for different types of $\mathrm{CdS}$ structures investigated. Analyzing values of fractional index $\alpha$ obtained in the range of $\alpha=$ 0.16-0.20 for different samples and conclusions about the structural properties of studied polycrystalline layers can be indirectly drawn. Additionally, the obtained values of $\alpha<$ 1 hint on dominance of the anomalous character of excess carrier transport within a disordered structure of CdS polycrystals investigated. It has been inferred that samples of the II-type and the III-type are structurally similar and are characterized by fractal factor of $f \cong 0.14$, and that samples of the I-type exhibit a distinguishably disordered structure with fractal factor $f \cong 0.17$.

\section{Summary}

In summary, the performed study of the CdS polycrystalline material layers by combining different techniques enabled us to separate three types of samples, associated with different density of dopants and carrier capture centres. Value of free-carrier density of the $n_{0}=1.5 \cdot 10^{13} \mathrm{~cm}^{-3}$ was estimated for junctions of II- and III-type, and it determines concentration of shallow impurities. For the I-type samples, equilibrium carrier density is significantly smaller; thus, junction appeared to be fully depleted without external applied voltage.

Two spectral bands of photoluminescence have been observed within all types of samples, a narrower spectral band of green luminescence peaked at $500 \mathrm{~nm}$ and a wider band of red luminescence with a peak at $700 \mathrm{~nm}$. The ratio between the green PL to the red PL intensities as $R_{\mathrm{PL}}=I_{\mathrm{G}-\mathrm{PL}} / I_{\mathrm{R}-\mathrm{PL}}$ has been obtained for the I-type CdS samples $R_{\text {PL-I-type }}<$ 1 , while these values are estimated to be $R_{\text {PL-II-type }} \geq 1$ 
and $R_{\text {PL-III-type }}>1.5$, respectively. The photoluminescence photoquenching effect has been ascribed to the microcrystals within polycrystalline CdS material. The PL-PQ effect implies a complicated interplay of defects in redistribution of the photopulse excited carriers. The confocal microscopy images of CdS polycrystalline layers for different type samples enabled us to conclude that the most homogeneous CdS layer is obtained for the II-type samples, deposited at $260^{\circ} \mathrm{C}$ for $75 \mathrm{~min}$. While, in III-type samples deposited at $260^{\circ} \mathrm{C}$ for $80 \mathrm{~min}$, the PL spectra show that G-PL within microcrystals prevails the traps ascribed R-PL.

The ratio of amplitudes $r_{\text {asti }}=U_{\text {as }} / U_{\text {in }}$, of the asymptotic component $U_{\text {as }}$ (extrapolated to $t=0$ ) to the initial MW-PC peak $U_{\text {in }}$, can be a measure for carrier densities decaying within microcrystals and intercrystallites volume of $\mathrm{CdS}$ polycrystal. The observed differences of MW-PC transients can be explained by larger volumes of micro-crystallites in II- and III-type samples. These observations are corroborated by microscopy imaging. Additionally, density of deep traps is the smallest in microcrystals of III-type samples. While most homogeneous distribution of microcrystals has been obtained for II-type samples.

A linearization of MW-PC transients can be performed by using a stretched exponent approximation, with different fractional index $\alpha$ values for separated types of CdS structures. The obtained values of $\alpha=0.16-0.20$ hint on dominance of the anomalous character of excess carrier transport within a disordered structure of CdS polycrystals investigated. It has been inferred that samples of the II-type and the III-type are structurally similar and characterized by a fractal factor of $f \cong 0.14$, and that samples of the I-type show a distinguishably disordered structure characterized by the fractal factor $f \cong 0.17$.

\section{Acknowledgments}

This study was funded from the European Community's social foundation under Grant agreement no. VP1-3.1-ŠMM08-K-01-004/KS-120000-1756.

\section{References}

[1] R. Blachnik and A. Müller, "The formation of $\mathrm{Cu}_{2} \mathrm{~S}$ from the elements-I: copper used in form of powders," Thermochimica Acta, vol. 361, no. 1-2, pp. 31-52, 2000.

[2] Z. Nan, X. Y. Wang, and Z. Zhao, "Formation of various morphologies of copper sulfides by a solvothermal method," Journal of Crystal Growth, vol. 295, no. 1, pp. 92-96, 2006.

[3] V. A. Smyntyna, V. A. Borschak, M. I. Kutalova, N. P. Zatovskaya, and A. P. Balaban, "Sensor based on a non-ideal heterojunction to indicate X-ray images," Semiconductor Physics, Quantum Electronics and Optoelectronics, vol. 7, no. 2, pp. 222223, 2004.

[4] F. Z. Nataren, D. Vassilevski, S. Chandrasekhar, B. Bouchikhi, and S. Martinuzzi, " $\mathrm{Cu}_{x} \mathrm{~S} / \mathrm{Cd}_{1-y} \mathrm{Zn}_{y} \mathrm{~S} / \mathrm{CdS}$ evaporated backwall bifilm solar cell," Thin Solid Films, vol. 90, no. 3, pp. L49L52, 1982.
[5] G. She, X. Zhang, W. Shi et al., "Template-free electrochemical synthesis of single-crystal CuTe nanoribbons," Crystal Growth and Design, vol. 8, no. 6, pp. 1789-1791, 2008.

[6] C. Naşcu, I. Pop, V. Ionescu, E. Indrea, and I. Bratu, "Spray pyrolysis deposition of CuS thin films," Materials Letters, vol. 32, no. 2-3, pp. 73-77, 1997.

[7] M. A. Korzhuev, "Dufour effect in superionic copper selenide," Physics of the Solid State, vol. 40, no. 2, pp. 217-219, 1998.

[8] J. Cardoso, O. Gomezdaza, L. Ixtlilco, M. T. S. Nair, and P. K. Nair, "Conductive copper sulfide thin films on polyimide foils," Semiconductor Science and Technology, vol. 16, no. 2, pp. 123-127, 2001.

[9] V. M. García, P. K. Nair, and M. T. S. Nair, "Copper selenide thin films by chemical bath deposition," Journal of Crystal Growth, vol. 203, no. 1, pp. 113-124, 1999.

[10] K. L. Chopra and S. R. Das, Thin Film Solar Cells, Plenum Press, New York, NY, USA, 1983.

[11] E. Bertran, J. L. Morenza, J. Esteve, and J. M. Codina, "Electrical properties of polycrystalline in-doped CdS thin films," Journal of Physics D, vol. 17, no. 8, pp. 1679-1685, 1984.

[12] A. Goldenblum, G. Popovici, E. Elena, A. Oprea, and C. Nae, "All-evaporation-processed $\mathrm{Cu}_{2} \mathrm{~S} / \mathrm{CdS}$ solar cells with improved characteristics," Thin Solid Films, vol. 141, no. 2, pp. 215-221, 1986.

[13] E. Aperathitis and C. G. Scott, "The use of vacuum evaporation for production of the $\mathrm{Cu}_{2} \mathrm{~S}$ absorber layer in thin-film CdS solar cells," Journal of Physics: Condensed Matter, vol. 1, pp. SB269SB270, 1989.

[14] E. Vanhoecke, M. Burgelman, and L. Anaf, "Reactive sputtering of large-area $\mathrm{Cu}_{2} \mathrm{~S} / \mathrm{CdS}$ solar cells," Thin Solid Films, vol. 144, no. 2, pp. 223-228, 1986.

[15] L. Soriano, M. Leon, F. Arjona, and E. G. Camarero, "On the photoconductivity of copper sulphide polycrystalline thin films," Solar Energy Materials, vol. 12, no. 2, pp. 149-155, 1985.

[16] M. Kundu, T. Hasegawa, K. Terabe, K. Yamamoto, and M. Aono, "Structural studies of copper sulfide films: effect of ambient atmosphere," Science and Technology of Advanced Materials, vol. 9, no. 3, Article ID 035011, 2008.

[17] T. Yamamoto, K. Tanaka, E. Kubota, and K. Osakada, "Deposition of copper sulfide on the surface of poly(ethylene terephthalate) and poly(vinyl alcohol) films in the aqueous solution to give electrically conductive films," Chemistry of Materials, vol. 5, no. 9, pp. 1352-1357, 1993.

[18] E. Gaubas, I. Brytavskyi, T. Čeponis, J. Kusakovskij, and G. Tamulaitis, "Barrier capacitance characteristics of CdS- $\mathrm{Cu}_{2} \mathrm{~S}$ junction structures," Thin Solid Films, vol. 531, pp. 131-136, 2013.

[19] E. Gaubas, I. Brytavskyi, T. Čeponis, V. Kalendra, and A. Tekorius, "Spectroscopy of deep traps in $\mathrm{Cu}_{2} \mathrm{~S}-\mathrm{CdS}$ junction structures," Materials, vol. 5, pp. 2597-2608, 2012.

[20] S. Martinuzzi, F. Cabane-Brouty, J. Gervais, and A. Mostavan, "Study of $\mathrm{Cu}_{2} \mathrm{~S}-\mathrm{CdZnS}$ photocells and $\mathrm{CdS}$ "spray" layers. II-first results on characterization of CdS spray layers," in Proceedings of the International Conference on Solar Electricity, pp. 303-308, Toulouse, France, March 1976.

[21] E. Gaubas, J. Vaitkus, E. Simoen, C. Claeys, and J. Vanhellemont, "Excess carrier cross-sectional profiling technique for determination of the surface recombination velocity," Materials Science in Semiconductor Processing, vol. 4, no. 1-3, pp. 125-131, 2001.

[22] E. Gaubas, T. Čeponis, S. Sakalauskas, A. Uleckas, and A. Velička, "Fluence dependent variations of barrier charging and generation currents in neutron and proton irradiated Si particle 
detectors," Lithuanian Journal of Physics, vol. 51, no. 3, pp. 230236, 2011.

[23] E. Gaubas, T. Ceponis, and J. Vaitkus, "Impact of generation current on the evaluation of the depletion width in heavily irradiated Si detectors," Journal of Applied Physics, vol. 110, no. 3, Article ID 033719, 7 pages, 2011.

[24] V. E. Lashkarev, A. V. Liubchenko, and M. K. Sheinkman, NonEquilibrium Processes in Photoconductors, Naukova Dumka, Kiev, Ukraine, 1981.

[25] B. Ray, II-VI Compounds, Pergamon Press, Oxford, UK, 1969.

[26] S. Juršenas, G. Kurilčik, and A. Žukauskas, "Luminescenceintensity kinetics due to nonradiative capture by multiphonon emission in highly excited CdS and CdSe crystals," Physical Review B, vol. 54, no. 23, pp. 16706-16713, 1996.

[27] J. Pankove, Optical Processes in Semiconductors, Prentice Hall, Upper Saddle River, NJ, USA, 1971.

[28] A. Rose, Concepts in Photoconductivity and Allied Problems, Interscience Publishers, 1963.

[29] L. Pavesi, "Influence of dispersive exciton motion on the recombination dynamics in porous silicon," Journal of Applied Physics, vol. 80, no. 1, pp. 216-225, 1996.

[30] E. Gaubas, S. Juršènas, S. Miasojedovas, J. Vaitkus, and A. Žukauskas, "Carrier and defect dynamics in photoexcited semiinsulating epitaxial GaN layers," Journal of Applied Physics, vol. 96, no. 8, pp. 4326-4333, 2004.

[31] S. Havlin and D. Ben-Avraham, "Diffusion in disordered media," Advances in Physics, vol. 51, no. 1, pp. 187-292, 2002.

[32] J. C. Phillips, "Stretched exponential relaxation in molecular and electronic glasses," Reports on Progress in Physics, vol. 59, no. 9, pp. 1133-1207, 1996.

[33] J. C. Phillips, "Slow dynamics in glasses: a comparison between theory and experiment," Physical Review B, vol. 73, no. 10, Article ID 104206, 6 pages, 2006.

[34] J. C. Phillips, "Microscopic aspects of stretched exponential relaxation (SER) in homogeneous molecular and network glasses and polymers," Journal of Non-Crystalline Solids, vol. 357, no. 22-23, pp. 3853-3865, 2011. 

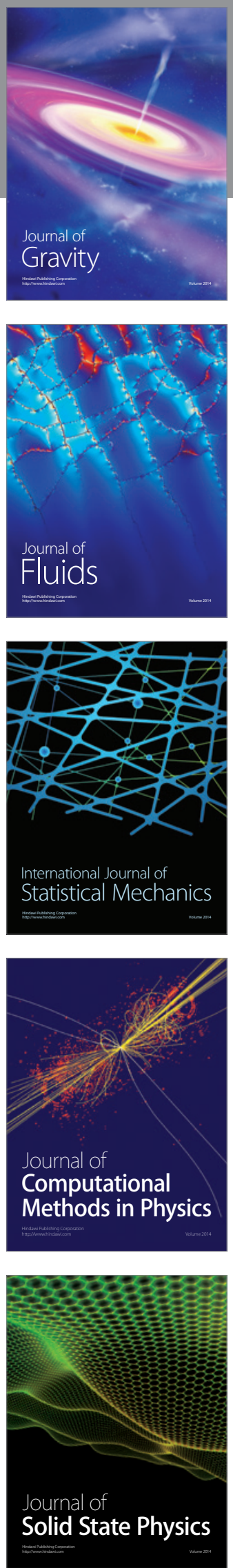

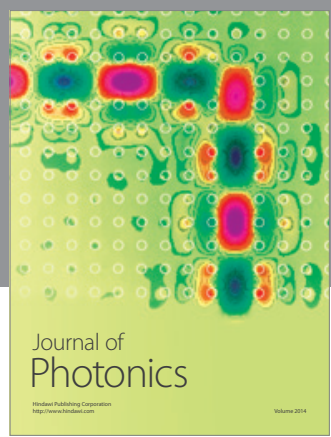

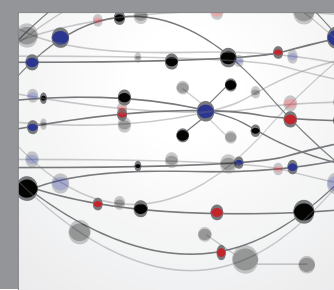

The Scientific World Journal

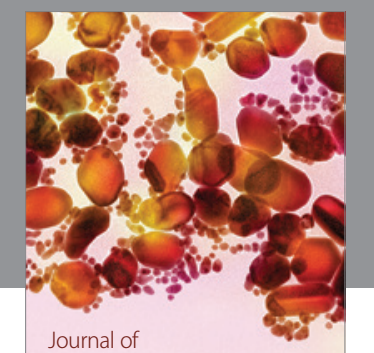

Soft Matter
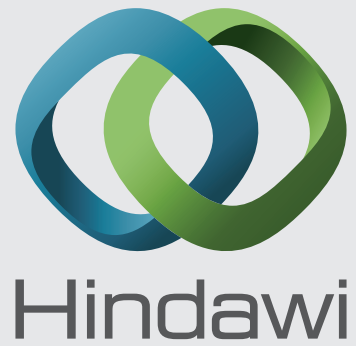

Submit your manuscripts at

http://www.hindawi.com
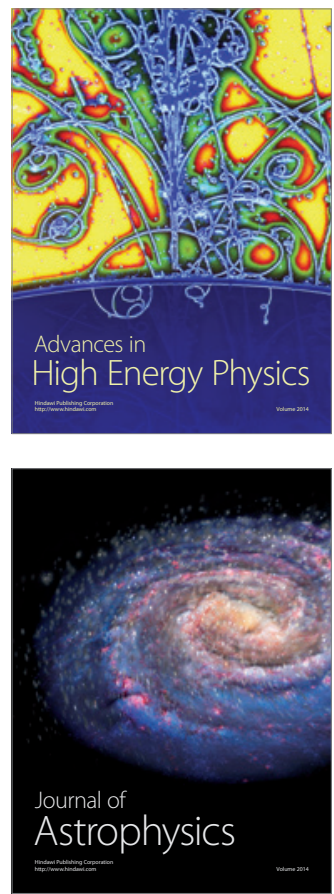
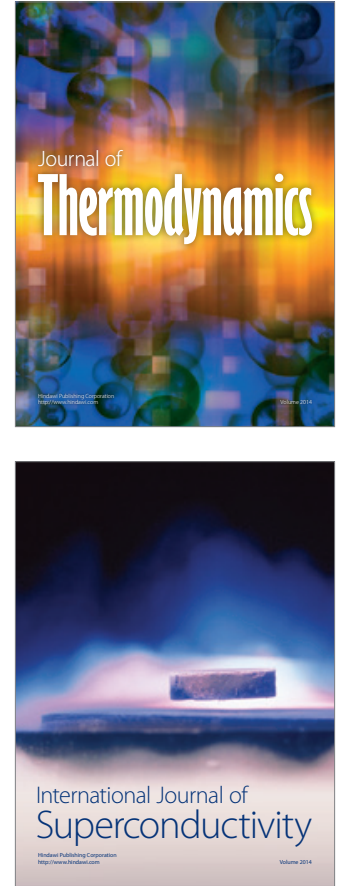
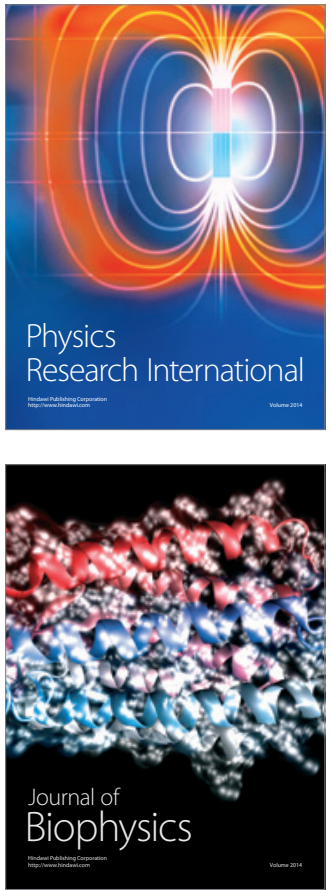
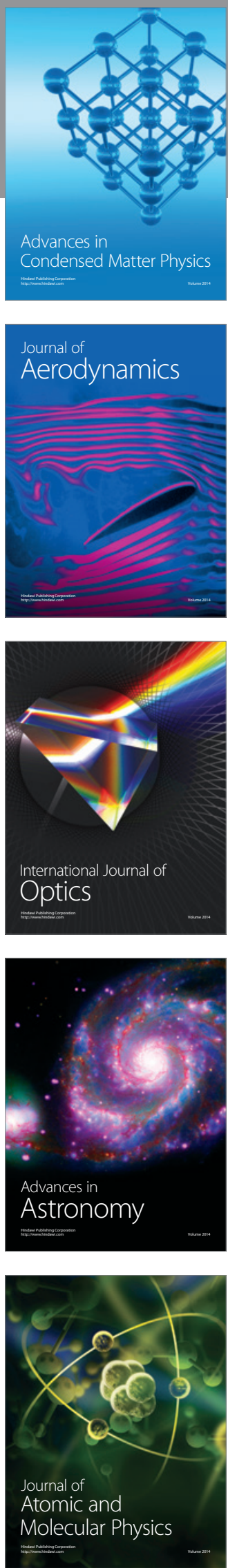